\title{
LIMITS OF LIMIT SETS II: GEOMETRICALLY INFINITE GROUPS
}

\author{
MAHAN MJ AND CAROLINE SERIES
}

\begin{abstract}
We show that for a strongly convergent sequence of purely loxodromic finitely generated Kleinian groups with incompressible ends, Cannon-Thurston maps, viewed as maps from a fixed base limit set to the Riemann sphere, converge uniformly. For algebraically convergent sequences we show that there exist examples where even pointwise convergence of Cannon-Thurston maps fails.
\end{abstract}

MSC classification: 30F40; 57M50

Keywords: Kleinian group, limit set, Cannon-Thurston map, geometrically infinite group

\section{Contents}

1. Introduction 2

2. Background 5

2.1. Kleinian groups 5

2.2. Balls and geodesics 5

2.3. The Cayley graph 6

2.4. Algebraic and Geometric Convergence 6

2.5. Scott cores

2.6. Relative Hyperbolicity and Electric Geometry 9

3. Cannon-Thurston Maps and Convergence Criteria $\quad 10$

3.1. Criteria for convergence 11

4. Strong Convergence 14

4.1. The bounded geometry case 14

4.2. Unbounded geometry 16

5. Algebraic limits and non-convergence of limit points 23

5.1. Brock's Examples 23

5.2. Absence of Uniform Convergence $\quad 28$

5.3. Pointwise non-convergence 30

Date: July 27, 2021.

Research of first author partially supported by CEFIPRA project 4301-1. 


\section{INTRODUCTION}

Given an isomorphism between Kleinian groups, a Cannon-Thurston map is a continuous equivariant map between their limit sets. It is by no means obvious that such a map always exists, however as the culmination of a long series of developments, it was shown in [37] that given a weakly type preserving (see below) isomorphism between any geometrically finite group $\Gamma$ and any Kleinian group $G$, a Cannon-Thurston map always exists.

This paper is the second of two dealing with convergence of Cannon-Thurston or $C T$-maps, considered as a sequence of continuous maps from the limit set of a fixed geometrically finite group to the sphere. The main questions addressed in both papers are:

(1) Does strong convergence of finitely generated Kleinian groups imply uniform convergence of $C T$-maps?

(2) Does algebraic convergence of finitely generated Kleinian groups imply pointwise convergence of $C T$-maps?

In the first paper [38] we dealt with the geometrically finite case by showing that both questions have a positive answer for a sequence of geometrically finite groups converging to a geometrically finite limit, provided that, in case (2), the geometric limit is also geometrically finite. As observed in [38], it is easy to see that if the groups converge algebraically but not strongly, then uniform convergence necessarily fails.

In the present paper we study the situation in which the limit group is geometrically infinite. We show that, in the absence of parabolics and with incompressible ends, the answer to (1) is always positive, but, in what is the most unexpected outcome of our investigations, we provide a counter example to (2) by exhibiting a sequence of geometrically finite groups converging algebraically but not strongly, for which the corresponding $C T$-maps fail to converge pointwise at a countable set of points. The class of limit groups in question are Brock's partially degenerate examples [9], described in more detail below. Thus our second main result answers in the negative the second part of Thurston's Problem 14 in his seminal paper [43]. In these examples, both the algebraic and geometric limits of the $G_{n}$ are geometrically infinite. We do not know whether there exist examples of non-convergence in which the algebraic limit is geometrically finite but the geometric limit is not.

Recall that an isomorphism $\rho: \Gamma \rightarrow G$ between Kleinian groups is strictly type preserving if $\rho(\gamma) \in G$ is parabolic if and only if $\gamma \in \Gamma$ is also parabolic; it is weakly type preserving if the 
image of any parabolic element is parabolic. Since $C T$-maps preserve fixed points, is easy to see that a necessary criterion for the existence of a $C T$-map $\hat{i}: \Lambda_{\Gamma} \rightarrow \Lambda_{G}$ between limit sets is that $\rho$ be weakly type preserving.

Our first main result, largely answering question (1), is:

Theorem A. Let $\Gamma$ be a geometrically finite Kleinian group without parabolics, which does not split as a free product. Let $\rho_{n}: \Gamma \rightarrow G_{n}$ be a sequence of strictly type preserving isomorphisms to geometrically finite Kleinian groups $G_{n}$, which converge strongly to a totally degenerate purely loxodromic Kleinian group $G_{\infty}=\rho_{\infty}(\Gamma)$. Then the sequence of CT-maps $\hat{i}_{n}: \Lambda_{\Gamma} \rightarrow \Lambda_{G_{n}}$ converges uniformly to $\hat{i}_{\infty}: \Lambda_{\Gamma} \rightarrow \Lambda_{G_{\infty}}$.

This result was proved by Miyachi [32] in the case in which $\Gamma$ is a surface group without parabolics and the injectivity radius is uniformly bounded below along the whole sequence. The condition that $\Gamma$ does not split as a free product is equivalent to requiring that all ends of the manifold $\mathbb{H}^{3} / \Gamma$ are incompressible, see [5].

Theorem A of [38], which is essentially the above result in the geometrically finite case, does not have any of the restrictions (absence of parabolics, not splitting as a free product, strictly type preserving, totally degenerate) imposed above. We introduce these restrictions largely because of technical issues concerning the model for the ends of the limit manifold $\mathbb{H}^{3} / G_{\infty}$. With a bit more work, similar techniques to those used here can be used to prove the theorem in the general case, see [40].

If the convergence is algebraic but not strong, then uniform convergence necessarily fails. This is an immediate consequence of Evans' theorem [17, 18] that the limit sets $\Lambda_{G_{n}}$ converge in the Hausdorff metric to the limit set of the geometric limit in the Hausdorff metric, see [38] for further discussion. As far as we know, the question of pointwise convergence in this situation has not previously been addressed. In answer to question (2) we have:

Theorem B. Let $\Gamma$ be a Fuchsian group for which $\mathbb{H}^{2} / \Gamma$ is a closed surface of genus at least 2. Then there exists a Kleinian group $G_{\infty}$, together with an isomorphism $\rho_{\infty}: \Gamma \rightarrow G_{\infty}=$ $\rho_{\infty}(\Gamma)$, and a sequence of representations $\rho_{n}: \Gamma \rightarrow G_{n}$ to geometrically finite groups converging algebraically to $G_{\infty}$, such that the sequence of CT-maps $\hat{i}_{n}: \Lambda_{\Gamma} \rightarrow \Lambda_{G_{n}}$ fails to converge pointwise to $\hat{i}_{\infty}$ at a countable set of points in $\Lambda_{\Gamma}$.

Implicit in the statement of Theorem $\mathrm{A}$ is the existence of the $C T$-map from $\Lambda_{\Gamma}$ to $\Lambda_{G_{\infty}}$. This result has a long history which we do not intend to repeat in detail here. The most general result, in which $G=G_{\infty}$ is an arbitrary torsion free non-elementary Kleinian group, can be found in [37]. The restricted case in which $\Gamma$ is a surface group and $G$ is singly or 
doubly degenerate, is the main result of [39], see Section 4 below. The original seminal case in which $M=\mathbb{H}^{3} / G$ is the cyclic cover of a 3-manifold fibering over the circle with fibers a closed surface is of course due to Cannon and Thurston [14]; this was extended to the case in which $G$ is a surface group with a lower bound on the lengths of loxodromics in [7] and [31], or more generally when $M$ is an arbitrary hyperbolic manifold with incompressible boundary in [34]. The older history in the case in which $G$ is geometrically finite is discussed in [38].

In [38] we introduced general criteria for uniform and pointwise convergence of $C T$-maps, called UEPP and EPP respectively. These compare the geometry of the obvious embedding of the Cayley graph of the base group $\Gamma$ into $\mathbb{H}^{3}$, to the corresponding embeddings for the groups $G_{n}, G_{\infty}$. The main work in this paper consists in verifying that these criteria hold (in the case of Theorem A) or understanding why they do not (in the case of Theorem B). After slightly reformulating the condition UEPP, we see that for the case of strictly type preserving maps of surface groups, the needed condition has essentially already been proved in [39]. In order to explain this, we give in Section 4.2 a brief outline of the relevant parts of the arguments in [39]. For the benefit of readers who have not gone through all of this previous work, which in turn depends heavily on the Minsky model of degenerate Kleinian groups, we preface this by briefly sketching in Section 4.1 how the argument goes in the case of groups of bounded geometry, thus reproving Miyachi's theorem [32]. Our proof in this case follows easily using the method explained in [31] and [36] and is independent of [39].

Let $R$ be a surface with boundary and $\Lambda$ a lamination on $R$. A complementary region in $R \backslash \Lambda$ is called a crown domain if it contains a component of $\partial R$. The counter example in Theorem B arises from Brock's examples of a sequence of quasi-Fuchsian groups $G_{n}$ converging algebraically but not strongly to a partially degenerate group $G_{\infty}$. More precisely, we prove the following, which immediately implies Theorem B:

Theorem C. Fix a closed hyperbolizable surface $S$ together with a separating simple closed curve $\sigma$, dividing $S$ into two pieces $L$ and $R$. Let $\alpha$ denote an automorphism of $S$ such that $\left.\alpha\right|_{L}$ is the identity and $\left.\alpha\right|_{R}=\chi$ is a pseudo-Anosov diffeomorphism of $R$ fixing the boundary $\sigma$. Let $X$ be a hyperbolic structure on $S$ and let $G_{n}$ be the quasi-Fuchsian group given by the simultaneous uniformization of $\left(\alpha^{n}(X), X\right)$. Let $G_{\infty}$ denote the algebraic limit of the sequence $G_{n}$, suitably normalized by a basepoint in the lift of the lower boundary $X$. Let $\hat{i}_{n}: \Lambda_{G_{0}} \rightarrow \Lambda_{G_{n}}$, $n \in \mathbb{N} \cup \infty$, be the corresponding CT-maps and let $\xi \in \Lambda_{G_{0}}$. Then $\hat{i}_{n}(\xi)$ converges to $\hat{i}_{\infty}(\xi)$ if and only if $\xi$ is not the endpoint of the lift to $\mathbb{H}^{2}$ of a boundary leaf, other than $\sigma$, of the crown domain of the unstable lamination of $\chi$, viewed as a lamination on the surface $R$. 
The outline of the paper is as follows. In Section 2 we set up background and notation, in particular reviewing briefly what we need from the theory of hyperbolic spaces and electric geometry in 2.6. These techniques are central in [39], and are also used here in the discussion of Theorem C.

In Section 3 we recall results from [38] on Cannon-Thurston maps, in particular we explain our convergence criterion UEPP. In Section 4 we prove Theorem $\mathrm{A}$. As discussed above, we first give a brief discussion of a proof in the case of bounded geometry, that is, when the injectivity radius of all manifolds in the sequence is uniformly bounded below. This is essentially Miyachi's theorem referred to above. We then turn to the general situation, outlining as we go the relevant steps in the proof for a single $C T$-map as in [39]. Finally in Section 5 we explain the counter examples to pointwise convergence, explaining the Brock examples and then proving Theorem C

Acknowledgments: This work was done in part while the first author was visiting Université Paris-Sud XI under the Indo-French collaborative programme ARCUS. He gratefully acknowledges their support and hospitality.

\section{BACKGROUND}

2.1. Kleinian groups. A Kleinian group $G$ is a discrete subgroup of $P S L_{2}(\mathbb{C})$. As such it acts as a properly discontinuous group of isometries of hyperbolic 3 -space $\mathbb{H}^{3}$, whose boundary we identify with the Riemann sphere $\hat{\mathbb{C}}=\mathbb{C} \cup \infty$. As in [38], all groups in this paper will be finitely generated and torsion free, so that $M=\mathbb{H}^{3} / G$ is a hyperbolic 3-manifold. The limit set $\Lambda_{G} \subset \hat{\mathbb{C}}$ is the set of accumulation points of any $G$-orbit.

A Kleinian group is geometrically finite if it has a fundamental polyhedron in $\mathbb{H}^{3}$ with finitely many faces; a group which is not geometrically finite is also called degenerate. The point of this paper is to investigate the extension of $[38$ to the degenerate case. We say a group is totally degenerate if it is not geometrically finite and $\Lambda_{G}=\hat{\mathbb{C}}$. The structure of degenerate groups has recently been elucidated by the work of Minsky et al. [29, 10] on the ending lamination theorem and the tameness theorem of Agol [1] and Calegari and Gabai [11]. This paper rests heavily on these results.

A Kleinian group $G$ is a surface group, if there is a hyperbolic surface $S$, together with a discrete faithful representation $\rho: \pi_{1}(S) \rightarrow G$. The corresponding manifold $\mathbb{H}^{3} / G$ is homeomorphic to $S \times \mathbb{R}$, see [5]. It is singly or doubly degenerate according as one or both of its ends are geometrically infinite with filling ending laminations.

2.2. Balls and geodesics. We will be working in hyperbolic space $\mathbb{H}^{n}$ for $n=2,3$. We denote the hyperbolic metric on $\mathbb{H}^{n}$ by $d_{\mathbb{H}}$ or occasionally $d_{\mathbb{H}^{n}}$; sometimes we explicitly use the ball 
model $\mathbb{B}$ with centre $O$ and denote by $d_{\mathbb{E}}$ the Euclidean metric on $\mathbb{B} \cup \hat{\mathbb{C}}$. For $P \in \mathbb{H}^{n}$, write $B(P ; R)$, or when needed $B_{\mathbb{H}}(P ; R)$ or even $B_{\mathbb{H}^{n}}(P ; R)$, for the hyperbolic ball centre $P$ and radius $R$. Let $\beta$ be a path in $\mathbb{H}^{n}$ with endpoints $X, Y$. We write $[\beta]$ or $[X, Y]$ for the $\mathbb{H}^{n}$-geodesic from $X$ to $Y$.

2.3. The Cayley graph. Let $G$ be a finitely generated Kleinian group with generating set $G^{*}=\left\{e_{1}, \ldots, e_{k}\right\}$. We assume throughout that $G^{*}$ is symmetric, in the sense that $g \in G^{*}$ if and only if $g^{-1} \in G^{*}$ for any $g \in G$. The Cayley graph $\mathcal{G} G$ of $G$ is the graph whose vertices are elements $g \in G$ and which has an edge between $g, g^{\prime}$ whenever $g^{-1} g^{\prime} \in G^{*}$. The graph metric $d_{G}$ is defined as the edge length of the shortest path between vertices so that $d_{G}\left(1, e_{i}\right)=1$ for all $i$, where 1 is the unit element of $G$. Let $|g|$ denote the word length of $g \in G$ with respect to $G^{*}$, so that $|g|=d_{G}(1, g)$. For $X \in \mathcal{G} G$, we denote by $B_{G}(X ; R) \subset \mathcal{G} G$ the $d_{G}$-ball centre $X$ and radius $R$.

Choose a basepoint $O_{G} \in \mathbb{H}^{3}$ which is not a fixed point of any element of $G$. One may if desired assume the basepoint is the centre $O$ of the ball model $\mathbb{B}$ as above. For simplicity, we do this throughout the paper unless indicated otherwise. Then $\mathcal{G} G$ is immersed in $\mathbb{H}^{3}$ by the map $j_{G}$ which sends $g \in G$ to $j_{G}(g)=g \cdot O$, and which sends the edge joining $g, g^{\prime}$ to the $\mathbb{H}^{3}$-geodesic joining $j_{G}(g), j_{G}\left(g^{\prime}\right)$. In particular, $j_{G}(1)=O$. Note that using the ball model of $\mathbb{H}^{3}$, the limit set $\Lambda_{G}$ may be regarded as the completion of $j_{G}(\mathcal{G} G)$ in the Euclidean metric $d_{\mathbb{E}}$ on $\mathbb{B} \cup \hat{\mathbb{C}}$.

2.4. Algebraic and Geometric Convergence. Let $\Gamma$ be a geometrically finite Kleinian group. A sequence of group isomorphisms $\rho_{n}: \Gamma \rightarrow P S L_{2}(\mathbb{C}), n=1,2 \ldots$ is said to converge to the representation $\rho_{\infty}: \Gamma \rightarrow P S L_{2}(\mathbb{C})$ algebraically if for each $g \in \Gamma, \rho_{n}(g) \rightarrow \rho_{\infty}(g)$ as elements of $P S L_{2}(\mathbb{C})$. The representations converge geometrically if $\left(G_{n}=\rho_{n}(G)\right)$ converges as a sequence of closed subsets of $P S L_{2}(\mathbb{C})$ to $G_{g} \subset P S L_{2}(\mathbb{C})$. Then $G_{g}$ is a Kleinian group called the geometric limit of $\left(G_{n}\right)$. The sequence $\left(\rho_{n}\right)$ converges strongly to $\rho_{\infty}(G)$ if $\rho_{\infty}(G)=G_{g}$ and the convergence is both geometric and algebraic. If a sequence of groups converge algebraically, they have a geometrically convergent subsequence, see [24] Theorem 4.4.3.

Alternatively, following Thurston [42], see also for example [12] Chapter 3, we say that a sequence of manifolds with base-frames $\left(M_{n}, \omega_{n}\right)$ converges geometrically (or in the $C^{\infty}$ Gromov-Hausdorff topology) to a manifold with base-frame $\left(M_{\infty}, \omega_{\infty}\right)$ if for each compact submanifold $C \subset M_{\infty}$ containing the base-frame $\omega_{\infty}$, there are smooth embeddings $\psi_{n}: C \rightarrow$ $M_{n}$ (for all sufficiently large $n$ ) which map base-frame to base-frame and such that $\psi_{n}$ converges to an isometry in the $C^{\infty}$-topology. Kleinian groups $G_{n}$ are said to converge geometrically 
to $G_{\infty}$ if the corresponding framed manifolds $\left(M_{n}=\mathbb{H}^{3} / G_{n}, \omega_{n}\right)$ converge geometrically to $\left(M_{\infty}=\mathbb{H}^{3} / G_{\infty}, \omega_{\infty}\right)$, where the base-frames $\omega_{n}, \omega_{\infty}$ are all the projection of a fixed base-frame in $\mathbb{H}^{3}$. The sequence $\left(\left(M_{n}, \omega_{n}\right)\right)$ converges strongly to $\left(M_{\infty}, \omega\right)$ if the convergence is geometric and in addition the convergence of $\left(\rho_{n}\right)$ to $\rho_{\infty}$ is algebraic. We remark that changing the basepoints in the above discussion may result in a different geometric limit.

The relation between these definitions is the following. Fix once and for all a standard base frame $\Omega$ in $\mathbb{H}^{3}$, with basepoint at the origin $O$ in the ball model of hyperbolic 3-space. Given a framed manifold $(M, \omega)$, there is a unique developing map $(\widetilde{M}, \tilde{\omega}) \rightarrow \mathbb{H}^{3}$ (where $\widetilde{M}$ is the universal cover of $M$ ) which sends a fixed lift $\tilde{\omega}$ of $\omega$ to $\Omega \in \mathbb{H}^{3}$. The induced holonomy homomorphism sends $\pi_{1}(M, o)$ to a discrete torsion free subgroup of $S L(2, \mathbb{C})$, where $o \in M$ is the basepoint of $\omega$. By for example [12] Theorem 3.2.9, this map is a homeomorphism with appropriate topologies, so that convergence of manifolds in the sense of Thurston is equivalent to geometric convergence in the first sense defined above, see for example [24] Chapter 4 or [23] Theorem 8.11. In particular, the map $\psi_{n}: C \rightarrow M_{n}$ is the projection to the quotient manifolds of a bi-Lipschitz embedding $\tilde{\psi}_{n}: B(O ; R) \rightarrow \mathbb{H}^{3}$ where $B(O ; R) \subset \mathbb{H}^{3}$ is a large ball whose projection to $\mathbb{H}^{3} / G_{\infty}$ contains $C$, see for example [3] Lemma 9.6.

2.5. Scott cores. Recall that a Scott core of a 3-manifold $V$ is a compact connected 3submanifold $K_{V}$ such that the inclusion $K_{V} \hookrightarrow V$ induces an isomorphism on fundamental groups. The Scott core is unique up to isotopy [25]. Note that in general, the Scott core may be much smaller than the convex core, even when the group is convex cocompact. We shall need the following relationship between the Scott core and the ends of $V$.

Lemma 2.1 (5] Proposition 1.3, 23] Theorem 4.126). Let $K_{V}$ be a Scott core of a 3-manifold $V$. There is a bijective correspondence between the ends of $V$ and boundary components of $V \backslash K_{V}$. Hence each component of $\partial K_{V}$ bounds a non-compact component of $V \backslash K_{V}$ and each of these components is an end of $V$.

Let $\rho_{n}: \Gamma \rightarrow P S L_{2}(\mathbb{C})$ be a sequence of representations converging strongly to $\rho_{\infty}$. Fixing the base-frames as the projections $\omega_{n}$ of the frame $\Omega$ in $\mathbb{H}^{3}$ to $M_{n}=\mathbb{H}^{3} / G_{n}, n \in \mathbb{N} \cup \infty$, we obtain a corresponding sequence of framed hyperbolic manifolds $\left(M_{n}, \omega_{n}\right)$ converging geometrically to $M_{\infty}=\mathbb{H}^{3} / G_{\infty}$. Set $N=\mathbb{H}^{3} / \Gamma$ and let $K_{N}$ be a Scott core of $N$, chosen such that the baseframe $\omega_{N}$ for $N$ has basepoint $o_{N} \in K_{N}$. The representations $\rho_{n}$ induce homotopy equivalences $\phi_{n}: K_{N} \rightarrow M_{n}$. We can lift $\phi_{n}$ to maps $\tilde{\phi}_{n}: \widetilde{K}_{N} \rightarrow \widetilde{M}_{n}$ for $n \in \mathbb{N} \cup \infty$ and note that by our choices that $\tilde{\phi}_{n}\left(\tilde{\omega}_{N}\right)$ converges to $\tilde{\phi}_{\infty}\left(\tilde{\omega}_{N}\right)$. 
In general the homotopy equivalences $\phi_{n}$ may not be homeomorphisms. However in the situation of strong convergence, the proof of [13] Proposition 3.3 or [3] Lemma 9.7, (see also Lemma 3.6 and the first part of the proof of Theorem A in [4]) gives:

Lemma 2.2. Let $\Gamma$ be a geometrically finite group and let $\rho_{n}$ be a sequence of discrete faithful representations of $\Gamma$ converging strongly to $\rho_{\infty}$. Let $\mathcal{K}=K_{M_{\infty}}$ be a compact core for $M_{\infty}=\mathbb{H}^{3} / \rho_{\infty}(\Gamma)$. Let $\psi_{n}: \mathcal{K} \rightarrow M_{n}$ be the bi-Lipschitz embeddings coming from the geometric convergence, inducing maps $\left(\psi_{n}\right)_{*}: \rho_{\infty}(\Gamma) \rightarrow \rho_{n}(\Gamma)$. Then for all large enough $n$, $\left(\psi_{n}\right)_{*}=\rho_{n} \circ \rho_{\infty}^{-1}$ and $\psi_{n}(\mathcal{K})$ is a compact core for $M_{n}=\mathbb{H}^{3} / \rho_{n}(\Gamma)$.

We remark that the hypotheses in [13] and [3] that all groups be purely hyperbolic, or indeed that the convergence be strictly type preserving, are not needed for this lemma. Also note that as remarked above, in general $\psi_{n}(\mathcal{K})$ may be much smaller than the convex core of $M_{n}$. If the convergence is not strong, Lemma 2.2 may fail even when the limit group is geometrically finite, as is shown by the well known Anderson-Canary examples [2].

Lemma 2.2 shows that, in the situation of strong convergence, we may take the homotopy equivalences $\phi_{n}$ to be homeomorphisms between Scott cores of the relevant groups. It also allows us to identify the ends of $M_{\infty}$ with the ends of the approximating groups. We have:

Corollary 2.3. Let $\Gamma$ be a geometrically finite group and let $\rho_{n}$ be a sequence of discrete faithful representations of $\Gamma$ converging strongly to $\rho_{\infty}$. Then, up to replacing $\Gamma$ by the group $G_{n_{0}}$ for some $n_{0} \in \mathbb{N}$, we can pick a Scott core $K$ of $\mathbb{H}^{3} / \Gamma$ such that there are bi-Lipschitz embeddings $\phi_{n}: K \rightarrow \mathbb{H}^{3} / G_{n}$ which induce $\rho_{n}$, and such that $\phi_{n}(K)$ is a Scott core of $M_{n}$ for $n \in \mathbb{N} \cup \infty$. Moreover suppose that $E$ is an end of $M_{\infty}$ and $U$ is the component of $M_{\infty} \backslash \phi_{\infty}(K)$ which is a neighborhood of $E$. Let $F=\partial U$ and let $U_{n}$ be the component of $M_{n} \backslash \phi_{n}(K)$ bounded by $\phi_{n} \phi_{\infty}^{-1}(F)$. Then $U_{n}$ is a neighborhood of an end $E_{n}$ of $M_{n}$ and we say that $U_{n}$ corresponds to $E$.

Proof. Set $\mathcal{K}=\phi_{\infty}(K)$. Take $n_{0}$ large enough that the conclusion of Lemma 2.2 applies. Replacing $N=\mathbb{H}^{3} / \Gamma$ by $M_{n_{0}}, \Gamma$ by $G_{n_{0}}$, and $\rho_{n}$ by $\rho_{n}^{\prime}=\rho_{n} \rho_{n_{0}}^{-1}$, we have a sequence of representations as before. The core of $M_{n_{0}}$ can be taken to be $\psi_{n_{0}}(\mathcal{K})$. Noting that $\rho_{n}^{\prime}$ is induced by $\psi_{n} \psi_{n_{0}}^{-1}: \psi_{n_{0}}(\mathcal{K}) \rightarrow M_{n}$, we can replace the homotopy equivalences $\phi_{n}: K_{N} \rightarrow M_{n}$ by the homeomorphisms $\psi_{n} \psi_{n_{0}}^{-1}: \psi_{n_{0}}(\mathcal{K}) \rightarrow \psi_{n}(\mathcal{K})$ between the cores of $M_{n_{0}}$ and $M_{n}, n>n_{0}$. These converge to the homeomorphism $\psi_{n_{0}}^{-1}: \psi_{n_{0}}(\mathcal{K}) \rightarrow \mathcal{K}$. In other words, we may as well assume that the homotopy equivalences $\phi_{n}$ are actually embeddings of the core $K_{N}$ of $N$ into $M_{n}, M_{\infty}$

The idea of the final statement follows [13] $\S 8$. That $U_{n}$ is a neighborhood of an end $E_{n}$ of $M_{n}$ follows from Lemma 2.1 . 
2.6. Relative Hyperbolicity and Electric Geometry. We summarize the facts we need on relative hyperbolicity and electric geometry. For further details, we refer the reader to [20, 6] see also [35] Section 3.

Let $(X, d)$ be a $\delta$-hyperbolic metric space, and let $\mathcal{H}$ be a collection of pairwise disjoint subsets. To electrocute $\mathcal{H}$ means to construct an auxiliary metric space $\left(X_{e l}, d_{e l}\right)$ in which the sets in $\mathcal{H}$ effectively have zero diameter, although for technical reasons it is preferable they have diameter 1 (or 2). Precisely, let $X_{e l}=X \bigcup_{H \in \mathcal{H}}(H \times[0,1])$ with $H \times\{0\}$ identified to $H \subset X$. We define the electric (pseudo)-metric $d_{e l}$ on $X_{e l}$ as follows. First equip $H \times[0,1]$ with the product metric and then modify this to a pseudo-metric by quotienting so that $H \times\{1\}$ is equipped with the zero metric. The metric on $H \times[0,1]$ is the path metric induced by horizontal and vertical paths. This means that in the space $\left(X_{e l}, d_{e l}\right)$, any two points in $H$ are at distance at most 2 .

Definition 2.4. [20, 6] Let $X$ be a metric space and $\mathcal{H}$ be a collection of mutually disjoint subsets. If $X_{e l}$ is also a hyperbolic metric space, then $X$ is said to be weakly hyperbolic relative to the collection $\mathcal{H}$.

The collection $\mathcal{H}$ is said to be uniformly separated if there exists $C>0$ such that $d\left(H_{i}, H_{j}\right) \geq$ $C$ for all $H_{i} \neq H_{j} \in \mathcal{H}$. It is uniformly quasi-convex if there exists $C>0$ such that if for any $H \in \mathcal{H}$ and for any points $x, x^{\prime} \in H$, any geodesic joining them lies within the $C$-neighborhood of $H$. It is mutually cobounded if there exists $C>0$ such that for all $H_{i} \neq H_{j} \in \mathcal{H}, \pi_{i}\left(H_{j}\right)$ has diameter less than $C$, where $\pi_{i}$ denotes a nearest point projection of $X$ onto $H_{i}$.

Lemma 2.5 ([6], [20] Proposition 4.6). Let $X$ be a hyperbolic metric space and $\mathcal{H}$ a collection of uniformly quasi-convex mutually cobounded uniformly separated subsets. Then $X$ is weakly hyperbolic relative to the collection $\mathcal{H}$.

A typical example is when $X$ is hyperbolic space $\mathbb{H}^{3}$ and $\mathcal{H}$ is the collection of lifts to $\mathbb{H}^{3}$ of the thin parts of a hyperbolic 3-manifold.

Following Farb, we need to understand some finer details of the relationship between geodesics in $X$ and in $X_{e l}$. Recall that $K$-quasi-geodesic in a metric space $Y$ is a $K$-quasiisometric embedding of an interval into $Y$, that is, a map $f:[a, b] \rightarrow Y$ such that

$$
\frac{1}{K}\left|t_{1}-t_{2}\right|-K \leq d_{Y}\left(f\left(t_{1}\right), f\left(t_{2}\right)\right) \leq K\left|t_{1}-t_{2}\right|+K
$$

for all $t_{1}, t_{2} \in[a, b]$. A quasi-geodesic is a path in $Y$ which is a $K$-quasi-geodesic for some $K>0$. If $X, \mathcal{H}$ gives rise to an electric space $\left(X_{e l}, d_{e l}\right)$, then an electric (quasi)-geodesic in $X$ is a path in $X$ which is a (quasi)-geodesic for the electric metric $d_{e l}$. 
We say that a path does not backtrack if it does not re-enter any $H \in \mathcal{H}$ after leaving it. Suppose that $\lambda$ is an electric quasi-geodesic in $\left(X_{e l}, d_{e l}\right)$ without backtracking and with endpoints $a, b \in X \backslash \mathcal{H}$. Keeping the endpoints $a, b$ fixed, replace each maximal subsegment of $\lambda$ lying within some $H \in \mathcal{H}$ by a hyperbolic $X$-geodesic with the same endpoints. The resulting connected path is called an electro-ambient quasi-geodesic in $X$, see Figure 1. The main result we need is:

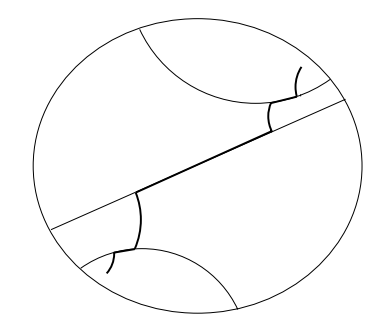

Figure 1. An electro-ambient quasi-geodesic.

Lemma 2.6 ([35] Lemma 3.7). Let $X$ be a hyperbolic metric space and let $\mathcal{H}$ be a collection of mutually cobounded uniformly separated uniformly quasi-convex sets. Let $\gamma$ be an electroambient quasi-geodesic with endpoints $a, b \in X \backslash \mathcal{H}$. Then $\gamma$ is a quasi-geodesic in $X$ and lies within bounded distance of any $X$-geodesic with the same endpoints.

In the case in which $X$ is hyperbolic space $\mathbb{H}^{3}$ and $\mathcal{H}$ is the collection of lifts to $\mathbb{H}^{3}$ of the thin parts of a hyperbolic 3-manifold, the proof of Lemma 2.6 is straightforward and was done from first principles in [38] Lemmas 7.15, 7.16.

Remark 2.7. One can introduce a further property of a metric space $X$ being strongly hyperbolic relative to a collection $\mathcal{H}$, see [35] Section 3 and also [6]. This condition concerns how paths penetrate the sets in $\mathcal{H}$. If $X$ is itself a $\delta$-hyperbolic space, then the conditions that the sets in $\mathcal{H}$ be mutually cobounded, uniformly separated and uniformly quasi-convex are enough to imply that $X$ is strongly hyperbolic relative to $\mathcal{H}$, see [20] $\S 4$ and [35] $\S 3$. Since all that we needed here is the result of Lemma 2.6, we do not digress to give the precise definition here.

\section{Cannon-Thurston Maps and Convergence Criteria}

Let $\Gamma$ be a Kleinian group and let $\rho: \Gamma \rightarrow P S L_{2}(\mathbb{C})$ with $\rho(\Gamma)=G$. Let $\Lambda_{\Gamma}, \Lambda_{G}$ be the corresponding limit sets. A Cannon-Thurston or CT-map is an equivariant continuous map $\hat{i}: \Lambda_{\Gamma} \rightarrow \Lambda_{G}$, that is, a map such that

$$
\hat{i}(g \cdot \xi)=\rho(g) \hat{i}(\xi) \text { for all } g \in \Gamma, \xi \in \Lambda_{\Gamma} .
$$


Recall from Section 2.3 the natural embedding $j_{\Gamma}$ of the Cayley graph of $\mathcal{G} \Gamma$ into $\mathbb{H}^{3}$. The $C T$-map $\hat{i}=\hat{i}(\rho)$ can also be defined as the continuous extension to $\Lambda_{\Gamma} \subset \partial \mathbb{H}^{3}$ of the obvious map $i: j_{\Gamma}(\mathcal{G} \Gamma) \rightarrow \mathbb{H}^{3}$ defined by $i\left(j_{\Gamma}(g)\right)=\rho(g) \cdot O$.

Suppose alternatively that $N$ is a geometrically finite manifold homotopy equivalent to another hyperbolic manifold $M$ and let $\phi: K_{N} \rightarrow M$ be a homotopy equivalence between a Scott core $K_{N}$ of $N$ and $M$. It is easy to see that $\hat{i}$ is an extension to $\partial \mathbb{H}^{3}$ of any lifting $\widetilde{\phi}: \widetilde{K}_{N} \rightarrow \widetilde{M}$, since any fixed orbit of the action of $G$ on $\tilde{\phi}\left(\tilde{K}_{N}\right)$ can serve as a substitute for the orbit of the basepoint $O$. Notice however that the careful discussion in 2.4 is needed to fix basepoints if we are dealing with a sequences of groups.

In [38] we reproved Floyd's results [21] on the existence of CT-maps for geometrically finite groups:

Theorem 3.1 ([38] Theorem 4.2). Let $\Gamma, G$ be finitely generated geometrically finite groups and let $\phi: \Gamma \rightarrow G$ be weakly type preserving isomorphism. Then the CT-map $\hat{i}: \Lambda_{\Gamma} \rightarrow \Lambda_{G}$ exists.

Note that the examples in [2] show that there exist geometrically finite non-cusped manifolds which are homotopic but not homeomorphic, see also Lemma 2.2. We deduce from the above that the $C T$-map between their limit sets nonetheless exists and is a homeomorphism.

3.1. Criteria for convergence. We now collect some criteria for the existence and convergence of $C T$-maps from [38].

Theorem 3.2 ([38] Theorem 4.1). Let $\rho: \Gamma \rightarrow G$ be a weakly type preserving isomorphism of finitely generated Kleinian groups and suppose that $\Gamma$ is geometrically finite. The CT-map $\Lambda_{\Gamma} \rightarrow \Lambda_{G}$ exists if and only if there exists a function $f: \mathbb{N} \rightarrow \mathbb{N}$, such that $f(N) \rightarrow \infty$ as $N \rightarrow \infty$, and such that whenever $\lambda$ is a $d_{\Gamma}$-geodesic segment lying outside $B_{\Gamma}(1 ; N)$ in $\mathcal{G} \Gamma$, the $\mathbb{H}^{3}$-geodesic joining the endpoints of $i\left(j_{\Gamma}(\lambda)\right)$ lies outside $B_{\mathbb{H}}(O ; f(N))$ in $\mathbb{H}^{3}$.

In [39], the criterion was used in an alternative form which involves geodesics in $\mathbb{H}^{3}$ for the domain group $\Gamma$ also. Recall that a geometrically finite group is convex cocompact if its convex core is compact. In this case we can take the Scott core to be the convex core.

Theorem 3.3. Let $\rho: \Gamma \rightarrow G$ be a strictly type preserving isomorphism of finitely generated Kleinian groups, and suppose that $\Gamma$ is convex cocompact. Suppose that $K_{N}$ is the convex core of $N=\mathbb{H}^{3} / \Gamma$ and that $\phi: K_{N} \rightarrow \mathbb{H}^{3} / G$ is a homotopy equivalence. Then the CT-map $\Lambda_{\Gamma} \rightarrow \Lambda_{G}$ exists if and only if there exists a non-negative function $f: \mathbb{N} \rightarrow \mathbb{N}$, such that $f(M) \rightarrow \infty$ as $M \rightarrow \infty$, and such that whenever $\lambda$ is a $\mathbb{H}^{3}$-geodesic segment lying outside $B(O ; M)$, the $\mathbb{H}^{3}$-geodesic $[\tilde{\phi}(\lambda)]$ lies outside $B(\tilde{\phi}(O) ; f(M))$, where $\tilde{\phi}$ is a fixed lift of $\phi$ to the obvious map from $\widetilde{K_{N}}$ (the convex hull of $\Lambda_{\Gamma}$ ) to $\mathbb{H}^{3}$. 
Note that to make a sensible statement here we need to insist that, in addition to being compact, the core $K_{N}$ of $N$ should also be convex. If $\Gamma$ is Fuchsian group corresponding to a closed surface, the main case in [39], then this is not an issue.

If $\Gamma$ is convex cocompact, then to compare geodesics in $\mathcal{G} \Gamma$ and $\mathbb{H}^{3}$, one can use that any

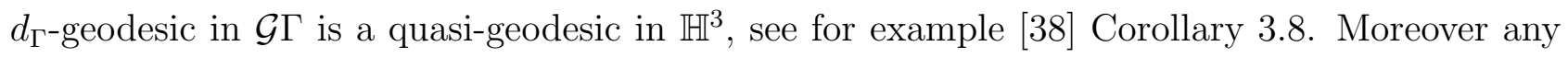
geodesic in $\mathbb{H}^{3}$ can be tracked at bounded distance by a path which is geodesic in $\mathcal{G} \Gamma$ (with bound depending only on $\Gamma$ ), see [38] Lemma 3.6. This shows the equivalence of the criteria in Theorems 3.2 and 3.3 in this case.

Remark 3.4. If $\Gamma$ is not convex cocompact then the comparison between geodesics in $\mathcal{G} \Gamma$ and $\mathbb{H}^{3}$ is not quite straightforward, since a geodesic path in $\mathcal{G} \Gamma$ can track round the boundary of a horosphere. This can be dealt with but requires more care. In Theorem $\mathrm{A}$ of this paper, $\Gamma$ is always convex cocompact, and in any case, we shall mainly stick to the first version of the criterion.

Now let $\Gamma$ be a fixed geometrically finite Kleinian group and suppose that $\rho_{n}: \Gamma \rightarrow P S L_{2}(\mathbb{C})$ is a sequence of discrete faithful weakly type preserving representations converging algebraically to $\rho_{\infty}: \Gamma \rightarrow P S L_{2}(\mathbb{C})$. Let $G_{n}=\rho_{n}(\Gamma), n \in \mathbb{N} \cup \infty$ and write $\Lambda_{n}$ for $\Lambda_{G_{n}}$. To normalize, we embed all the Cayley graphs with the same base-point $O=O_{G_{n}}$ for all $n$ and set $j_{n}(g)=$ $\rho_{n}(g) \cdot O, g \in \mathcal{G} \Gamma$. Define $i_{n}: j_{\Gamma}(\mathcal{G} \Gamma) \rightarrow \mathbb{H}^{3}$ by $i_{n}\left(j_{\Gamma}(g)\right) \mapsto \rho_{n}(g) \cdot O, g \in \mathcal{G} \Gamma$, so that the $C T$-map $\hat{i}_{n}: \Lambda_{\Gamma} \rightarrow \Lambda_{n}$ is the continuous extension of $i_{n}$ to $\Lambda_{\Gamma}$. Assuming they exist, we say that the $C T$-maps $\hat{i}_{n}: \Lambda_{\Gamma} \rightarrow \Lambda_{n}$ converge uniformly (resp. pointwise) to $\hat{i}_{\infty}$ if they do so as

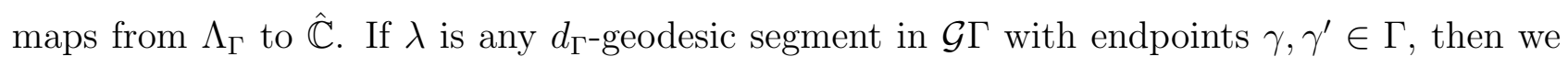
also write $[j(\lambda)]$ for the $\mathbb{H}^{3}$-geodesic $\left[j(\gamma), j\left(\gamma^{\prime}\right)\right]$.

In [38] we introduced two properties UEP (Uniform Embedding of Points) and UEPP (Uniform Embedding of Pairs of Points) of the sequence $\left(\rho_{n}\right)$. The first was shown in [38] to be equivalent to strong convergence, and the second is our criterion for uniform convergence of $C T$-maps. We summarise these definitions and results here.

Definition 3.5. Let $\rho_{n}: \Gamma \rightarrow G_{n}$ be a sequence of weakly type preserving isomorphisms of Kleinian groups. Then $\left(\rho_{n}\right)$ is said to satisfy UEP if there exists a non-negative function $f: \mathbb{N} \rightarrow \mathbb{N}$, with $f(N) \rightarrow \infty$ as $N \rightarrow \infty$, such that for all $g \in \Gamma, d_{\Gamma}(1, g) \geq N$ implies $d_{\mathbb{H}}\left(\rho_{n}(g) \cdot O, O\right) \geq f(N)$ for all $n \in \mathbb{N}$.

Definition 3.6. Let $\rho_{n}: \Gamma \rightarrow G_{n}$ be a sequence of weakly type preserving isomorphisms of Kleinian groups. Then $\left(\rho_{n}\right)$ satisfies UEPP if there exists a function $f^{\prime}: \mathbb{N} \rightarrow \mathbb{N}$, such that $f^{\prime}(N) \rightarrow \infty$ as $N \rightarrow \infty$, and such that whenever $\lambda$ is a $d_{\Gamma}$-geodesic segment lying outside $B_{\Gamma}(1 ; N)$ in $\mathcal{G} \Gamma$, the $\mathbb{H}^{3}$-geodesic $\left[j_{n}(\lambda)\right]$ lies outside $B_{\mathbb{H}}\left(O ; f^{\prime}(N)\right)$ for all $n \in \mathbb{N}$. 
Proposition 3.7 ([38] Proposition 5.3). Suppose that a sequence of discrete faithful weakly type preserving representations $\left(\rho_{n}: \Gamma \rightarrow P S L_{2}(\mathbb{C})\right)$ converges algebraically to $\rho_{\infty}$. Then $\left(\rho_{n}\right)$ converges strongly if and only if it satisfies UEP.

Theorem 3.8 ([38] Theorem 5.7). Let $\Gamma$ be a geometrically finite Kleinian group and let $\rho_{n}$ : $\Gamma \rightarrow G_{n}$ be weakly type preserving isomorphisms to Kleinian groups. Suppose that $\rho_{n}$ converges algebraically to a representation $\rho_{\infty}$. Then if $\left(\rho_{n}\right)$ satisfies UEPP, the CT-maps $\hat{i}_{n}: \Lambda_{\Gamma} \rightarrow \Lambda_{n}$ converge uniformly to $\hat{i}_{\infty}$. If $\Gamma$ is non-elementary, the converse also holds.

Inverting the function $f^{\prime}$ in the definition of UEPP gives a slight modification of the criterion in Theorem 3.8 convenient to our purposes. Note that in the statement which follows, we do not need to assume that $f_{1}(N) \rightarrow \infty$ as $N \rightarrow \infty$.

Corollary 3.9. Let $\Gamma$ be a geometrically finite Kleinian group and let $\rho_{n}: \Gamma \rightarrow G_{n}$ be weakly type preserving isomorphisms to Kleinian groups. Suppose that $\rho_{n}$ converges algebraically to a representation $\rho_{\infty}$ and that there exists a function $f_{1}: \mathbb{N} \rightarrow \mathbb{N}$ such that for any $L \in \mathbb{N}$, whenever $\lambda$ is a $d_{\Gamma}$-geodesic segment lying outside $B_{\Gamma}\left(1 ; f_{1}(L)\right)$ in $\mathcal{G} \Gamma$, the $\mathbb{H}^{3}$-geodesic $\left[j_{n}(\lambda)\right]$ lies outside $B_{\mathbb{H}}(O ; L)$ for all $n \in \mathbb{N}$. Then the CT-maps $\hat{i}_{n}: \Lambda_{\Gamma} \rightarrow \Lambda_{n}$ converge uniformly to $\hat{i}_{\infty}$

Proof. In view of Theorem 3.8, it is enough to see the given condition implies UEPP. We do this by inverting the function $f_{1}$. Precisely, say $L^{\prime}>L$. If $\lambda$ is a $d_{\Gamma}$-geodesic segment lying outside $B_{\Gamma}\left(1 ; f_{1}\left(L^{\prime}\right)\right)$ in $\mathcal{G} \Gamma$, the $\mathbb{H}^{3}$-geodesic $\left[j_{n}(\lambda)\right]$ lies outside $B_{\mathbb{H}}\left(O ; L^{\prime}\right)$ and hence certainly outside $B_{\mathbb{H}}(O ; L)$. Thus we may modify $f_{1}$ if needed so that $f_{1}\left(L^{\prime}\right)>f_{1}(L)$. Hence without loss of generality using an inductive argument we may assume that $f_{1}$ is strictly increasing, so that in particular $f_{1}(N) \rightarrow \infty$ as $n \rightarrow \infty$.

Now given $N \in \mathbb{N}$ define $f^{\prime}(N)=L$, where $L$ is the unique positive integer such that $N \in\left[f_{1}(L), f_{1}(L+1)\right)$. Note that with this definition, $f^{\prime}(N) \rightarrow \infty$ as $N \rightarrow \infty$.

Suppose given $N$ and that $f^{\prime}(N)=L$. We are given that whenever $\lambda$ is a $d_{\Gamma^{-}}$geodesic segment lying outside $B_{\Gamma}\left(1 ; f_{1}(L)\right)$ in $\mathcal{G} \Gamma$, the $\mathbb{H}^{3}$-geodesic $\left[j_{n}(\lambda)\right]$ lies outside $B_{\mathbb{H}}(O ; L)$ for all

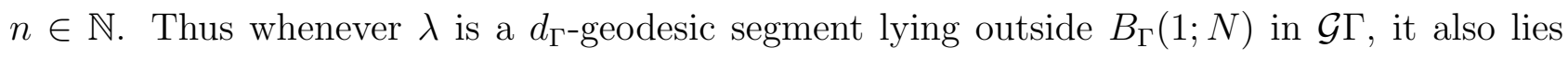
outside $B_{\Gamma}\left(1 ; f_{1}(L)\right)$ and hence the $\mathbb{H}^{3}$-geodesic $\left[j_{n}(\lambda)\right]$ lies outside $B_{\mathbb{H}}(O ; L)=B_{\mathbb{H}}\left(O ; f^{\prime}(N)\right)$ for all $n \in \mathbb{N}$. This is exactly the condition UEPP.

Applying a similar inversion to Theorem 3.2 we obtain immediately:

Corollary 3.10. Let $\Gamma$ be a geometrically finite Kleinian group and let $\rho: \Gamma \rightarrow G$ be a weakly type preserving isomorphism to a non-elementary Kleinian group $G$ for which a CT-map exists. 
Then there exists a function $f_{1}: \mathbb{N} \rightarrow \mathbb{N}$ such that for any $L \in \mathbb{N}$, whenever $\lambda$ is a $d_{\Gamma}$-geodesic segment lying outside $B_{\Gamma}\left(1 ; f_{1}(L)\right)$ in $\mathcal{G} \Gamma$, the $\mathbb{H}^{3}$-geodesic $\left.[j(\lambda))\right]$ lies outside $B_{\mathbb{H}}(O ; L)$.

\section{Strong Convergence}

In this section we prove Theorem A. To set the scene, we begin with a brief discussion in the case of strong convergence and bounded geometry, that is, when the injectivity radii of all manifolds in the sequence are uniformly bounded below. We then turn to the general situation of Theorem A, outlining as we go the relevant steps in the proof for a single CT-map as in [39].

4.1. The bounded geometry case. In this section, which is not necessary for the general result, we illustrate how to use the criteria of Section 3 to prove Theorem A in the case of a singly or doubly degenerate surface group with bounded geometry. This is essentially Miyachi's result [32]. The brief sketch below is a reprise of the first author's original arguments in [31, 36]. This sketch may also be useful to clarify the flow of the arguments from [39] which are the basis of our proof of Theorem A.

Recall that if $M$ is a hyperbolic 3-manifold, the injectivity radius $r(M ; x)$ of $M$ at $x \in M$ is $r / 2$ where $r$ is the length of the shortest loop based at $x$. The manifold is said to have bounded geometry if there exists $a>0$ such that $r(M ; x) \geq a$ for all $x \in M$; in this case the injectivity radius of $M$ is $\inf _{x \in M} r(M, x)$.

Theorem 4.1. Let $\Gamma$ be a Fuchsian group such that $\mathbb{H}^{2} / \Gamma$ is a closed hyperbolic surface. Let $\rho_{n}: \Gamma \rightarrow G_{n}$ be a sequence of strictly type-preserving isomorphisms to geometrically finite groups $G_{n}$, which converge strongly to a singly or doubly degenerate surface group $G_{\infty}=\rho_{\infty}(\Gamma)$. Suppose moreover that the injectivity radii of $M_{n}$ are uniformly bounded below by some a $>0$ for $n \in \mathbb{N} \cup \infty$. Then the sequence of CT-maps $\hat{i}_{n}: \Lambda_{\Gamma} \rightarrow \Lambda_{G_{n}}$ converges uniformly to $\hat{i}_{\infty}: \Lambda_{\Gamma} \rightarrow \Lambda_{G_{\infty}}$.

Proof. It is sufficient to show that the sequence $\left(\rho_{n}\right)$ satisfies UEPP. That is, we have to show that there exists a function $f^{\prime}: \mathbb{N} \rightarrow \mathbb{N}$ such that $f^{\prime}(N) \rightarrow \infty$ as $N \rightarrow \infty$ and such that

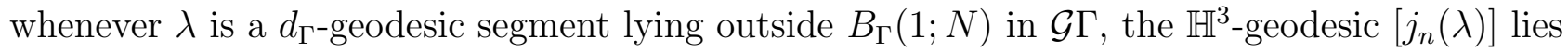
outside $B_{\mathbb{H}}\left(O ; f^{\prime}(N)\right)$ for all $n \in \mathbb{N}$.

By [31] Theorem 4.7, the Cannon-Thurston map exists for the group $G_{\infty}$. Hence, by Theorem 3.2, there exists a non-negative function $f: \mathbb{N} \rightarrow \mathbb{N}$, such that $f(N) \rightarrow \infty$ as $N \rightarrow \infty$, and such that whenever $\lambda$ is a $d_{\Gamma}$-geodesic segment lying outside $B_{\Gamma}(1 ; N)$ in $\mathcal{G} \Gamma$, the $\mathbb{H}^{3}$-geodesic joining the endpoints of $i_{\infty}\left(j_{\Gamma}(\lambda)\right)$ lies outside $B_{\mathbb{H}}\left(O_{G} ; f(N)\right)$ in $\mathbb{H}^{3}$. What we have to do is to show that the same function $f$ works uniformly for the representations $\rho_{n}$ for all sufficiently large $n$. 
Finding the function $f$ in [31] was based on the following construction. For simplicity, suppose that $G_{\infty}$ is doubly degenerate; the singly degenerate case is similar. Let $S$ be a topological surface homeomorphic to $\mathbb{H}^{2} / \Gamma$. By results of Minsky [27, 28], one can pick a sequence of pleated surface maps $S \rightarrow S_{n} \subset M_{\infty}=\mathbb{H}^{3} / G_{\infty}, n \in \mathbb{Z}$, such that the distance between $S_{n}, S_{n+1}$ is uniformly bounded above and below, and such that, with respect to the induced hyperbolic metrics on the $S_{n}$, there are uniformly bounded quasi-isometries $S_{n} \rightarrow S_{n+1}$. One deduces that the universal cover $\widetilde{M}_{\infty}$ of $M_{\infty}$ is quasi-isometric to a 'tree' of Gromov hyperbolic metric spaces. This is a Gromov hyperbolic space $\mathcal{X}$ equipped with a map $P$ onto a simplicial tree $\mathcal{T}$, which in the case of a doubly degenerate surface group can be taken to be the tree whose vertices are the integers, with a unit length edge joining $n$ to $n+1, n \in \mathbb{Z}$. The inverse image of each vertex is itself a Gromov hyperbolic space; these spaces are uniformly properly embedded into $\mathcal{X}$. Moreover for each pair of adjacent vertices $v, v^{\prime}$, there is a quasiisometry between the spaces $P^{-1}(v), P^{-1}\left(v^{\prime}\right)$, again assumed to have quasi-isometry constants uniform over vertices $v$.

In the present case, each space $P^{-1}(n)$ is quasi-isometric to the universal cover of $S_{n}$. In particular the map $P: P^{-1}(0) \rightarrow \mathcal{X}$ should be thought of as the lift to universal covers of the map $S_{0} \rightarrow M_{\infty}$. Since $S_{0}=\mathbb{H}^{2} / \Gamma$ is by assumption a closed surface, its universal cover is quasi-isometric to the Cayley graph of $\Gamma$. If we assume that the lift $O \in \mathbb{H}^{2}$ of the basepoint $o \in S_{0}$ maps to the lifted basepoint $O \in \mathbb{H}^{3}=\widetilde{M}_{\infty}$, then we can replace a geodesic $\lambda$ as in the statement of the theorem with a geodesic in the hyperbolic space $P^{-1}(0)$, see the comments following Theorem 3.3 .

We have to compare $\lambda$ with the hyperbolic geodesic $[\lambda]$ in $\widetilde{M}=\mathbb{H}^{3}$ joining its endpoints. The key idea is to construct a 'ladder' $\mathcal{L}_{\lambda} \subset \mathcal{X}$ by flowing $\lambda$ up the levels in $\mathcal{X}$ using the quasiisometries between the levels, see [31] for details. By constructing a coarse Lipschitz projection from $\mathcal{X}$ to $\mathcal{L}_{\lambda}$, it is shown that $\mathcal{L}_{\lambda}$ is uniformly quasi-convex in $\mathcal{X}$. That is, there exists $k>0$ such that any $\mathcal{X}$-geodesic with ends in $\mathcal{L}_{\lambda}$ lies within distance $k$ of $\mathcal{L}_{\lambda}$. A short argument given in the proof of [31] Theorem 3.10 shows that this is sufficient to construct the required function $f$ for the manifold $M$.

This function $f$ depends on the quasi-isometry between $\widetilde{M}$ and $\mathcal{X}$, and it is not hard to see by inspection that the constants depend only on the injectivity radius of $M$. The constant $k$ has a similar dependence. Thus if we have a family of manifolds all of which have the same lower bound on injectivity radii, the same function $f$ works simultaneously for all $M_{n}$ and the criterion of Corollary 3.9 is satisfied.

The problem with directly extending this proof to the situation of unbounded geometry, is that it requires a ladder and projection whose constants depend on the injectivity radius of 
the whole end. This is clearly not possible in the case of unbounded geometry. However we note that the same methods can be extended to the case of a surface with punctures, see [36] Section 5.5. (Essentially, this uses similar arguments about crossing horoballs to those in [38].) This gives the following result which we use in the proof of Proposition 5.12 in Section 5 .

Theorem 4.2. Let $\Gamma$ be a Fuchsian group such that $\mathbb{H}^{2} / \Gamma$ is a finite area hyperbolic surface. Let $\rho_{n}: \Gamma \rightarrow G_{n}$ be a sequence of strictly type-preserving isomorphisms to geometrically finite groups $G_{n}$, which converge strongly to a singly or doubly degenerate surface group $G_{\infty}=\rho_{\infty}(\Gamma)$. Suppose moreover that the injectivity radii of $M_{n}$ outside cusps are uniformly bounded below for $n=1,2, \ldots, \infty$. Then the sequence of CT-maps $\hat{i}_{n}: \Lambda_{\Gamma} \rightarrow \Lambda_{G_{n}}$ converges uniformly to $\hat{i}_{\infty}: \Lambda_{\Gamma} \rightarrow \Lambda_{G_{\infty}}$.

4.2. Unbounded geometry. Our proof of Theorem $\mathrm{A}$ is based on the method in [39], which effectively verifies the condition of Theorem 3.2 for a single group. To show that UEPP holds for a sequence converging strongly to such a limit, we need to examine the argument carefully to understand the dependence of the constants on the limit group. We simplify by explaining the first part of the proof in the case of surface groups, discussing extensions to general manifolds with incompressible boundary later.

The proof of Theorem A follows very closely that of the main result of [39], which can be roughly stated as:

Theorem 4.3 ([39] Theorem 7.1). Cannon-Thurston maps exist for simply or doubly degenerate surface Kleinian groups without cusps.

The actual result on which we base the proof of Theorem A is [39] Corollary 6.13, which we restate in an equivalent formulation as Lemma 4.7 below. To give more insight into what is involved, we begin by sketching the relevant parts of the proof of Theorem 4.3 . For this we first require a brief digression on split geometry as introduced in [39], which we do in 4.2.1. In 4.2 .2 we sketch the proof of Theorem 4.3. In 4.2.3 we explain the main technical result we use, Corollary 4.10. Finally in 4.2 .4 we prove Theorem $\mathrm{A}$

4.2.1. Split geometry. Let $S$ be a hyperbolizable surface and let $M$ be a manifold homeomorphic to $S \times \mathcal{I}$ where $\mathcal{I} \subset \mathbb{R}$ is an interval either finite or infinite. To say that $M$ has split geometry means, roughly speaking, that it is made by gluing together a succession (finite or infinite) of so-called split blocks. These are blocks each of which is homeomorphic to $S \times[0,1]$, split by annuli round Margulis tubes corresponding to short curves.

A split subsurface $S^{s}$ of a hyperbolic surface $S$ is a (possibly disconnected) proper subsurface with boundary, whose components are all essential and non-annular, and whose complement in 
$S$ is a non-empty family of non-homotopic annuli which are $k$-neighborhoods of non-peripheral geodesics on $S$. Moreover $S^{s}$ is required to have bounded geometry, in the sense that there exists some universal $\epsilon_{0}>0$ such that each boundary component of $S^{s}$ is of length $\epsilon_{0}$.

A split block $B^{s} \subset B=S \times[0,1]$ is a topological product $S^{s} \times[0,1]$ for some split subsurface $S^{s}$ of $S$, with the qualification that its upper and lower boundaries are only required to be split sub-surfaces of $S^{s}$. Split blocks are glued along their boundaries to build up model manifolds in the spirit of [29]. A split component is a connected component of a split block, see [39] following Definition 4.11 .

Suppose $\mathfrak{M}$ is a model manifold obtained by gluing finitely or infinitely many split blocks along their boundaries. Section 4.3 in [39] introduces a so-called graph metric $d_{\text {graph }}$ on the universal cover $\widetilde{\mathfrak{M}}$. (This metric is denoted $d_{G}$ in [39]; the notation here is used to distinguish it from the word metric on the Cayley graph.) Roughly speaking $d_{\text {graph }}$ is obtained by first electrocuting the lifts of Margulis tubes in each split block, and then by electrocuting the lifts of connected components of each split block. A (Gromov) hyperbolic manifold is said to be of split geometry, see [39] Definition 4.31, if each split component is quasi-convex (not necessarily uniformly) in the hyperbolic metric on $\widetilde{\mathfrak{M}}$ and if in addition, the hyperbolic convex hull of the universal cover of any split component has uniformly bounded diameter in the graph metric $d_{\text {graph }}$. This uniform bound is called the graph quasi-convexity constant and plays a crucial role in the discussion.

Given a singly or doubly degenerate hyperbolic manifold $M$ whose fundamental group is a surface group, a large part of the work in [39] is to construct a model manifold $\mathfrak{M}$ of split geometry whose universal cover $\widetilde{\mathfrak{M}}$ is bi-Lipschitz homeomorphic to the universal cover $\widetilde{M}$ of $M$. The model $\mathfrak{M}$ is made by consistently gluing finitely or infinitely many split blocks $B_{i}$ so that $B_{i-1}$ is glued to $B_{i}$ along their common boundary split surface $S_{i}$. The existence of the sequence of split level surfaces and split blocks exiting the end is a consequence of the Minsky model [29] for a degenerate end. The detailed construction is intricate and involves a careful selection of the split level surfaces using the Minsky hierarchy, see [39] especially $\S 3$ and $\S 4$ for details.

Theorem 4.1 ([39] Theorem 4.32). The hyperbolic manifold corresponding to any singly or doubly degenerate surface group without accidental parabolics is bi-Lipschitz homeomorphic to a model manifold with split geometry.

The part of this result which asserts uniform graph quasi-convexity of the blocks is [39] Proposition 4.23. We note the point, key for our purposes here, that the graph quasi-convexity constant is a combinatorial quantity which depends only on the topological convexity of the 
surface defining the end, and is thus also uniform across all degenerate ends of any hyperbolic manifold defined by the same topological surface $S$.

4.2.2. Rough sketch of Theorem 4.3. Let $S=\mathbb{H}^{2} / \Gamma$ be a compact hyperbolic surface and let $\rho: \Gamma \rightarrow G$ be a type preserving isomorphism to a singly or doubly degenerate surface group $G$. The criterion used in [39] to prove the existence of a CT-map for $\rho$ is that given in Theorem 3.2. but it will be convenient for our purposes to use the alternative formulation of Corollary 3.10 . In view of Theorem 4.1, we may work either with $M=\mathbb{H}^{3} / G$, or with a quasi-isometric model manifold of split geometry $\mathfrak{M}$. Lifting to universal covers, we obtain an identification of the universal cover $\widetilde{\mathfrak{M}}$ of $\mathfrak{M}$ with $\mathbb{H}^{3}$, and in particular we can identify a basepoint $O \in \mathbb{H}^{3}$ with a point, also denoted $O$, in $\widetilde{\mathfrak{M}}$.

Here is the statement we need:

Proposition 4.4. Let $G$ be a totally degenerate surface group corresponding to a strictly type preserving representation $\rho: \pi_{1}(S) \rightarrow G$ where $S$ is a closed surface as above, and let $\mathfrak{M}$ be a model manifold of split geometry corresponding to $M=\mathbb{H}^{3} / G$. Let $B_{0} \subset S_{0} \times[0,1]$ be a fixed base block and let $\phi: S \rightarrow \mathfrak{M}$ be the embedding which identifies $S$ with $S_{0} \times\{0\} \subset B_{0}$. Fix a basepoint $O \in \widetilde{S}$ in the universal cover $\widetilde{S}=\mathbb{H}^{2}$. Denote by $\widetilde{\phi}$ the lift of $\phi$ such that $\widetilde{\phi}(O)$ is the basepoint $O \in \mathbb{H}^{3}=\widetilde{\mathfrak{M}}$.

Then for any $L \in \mathbb{N}$, there exists $f(L) \in \mathbb{N}$, such that whenever $\lambda$ is a geodesic segment in $\left(\widetilde{S}, d_{S}\right)$ lying outside an $f(L)$-ball around $O \in \widetilde{S}$ (where $d_{S}$ is the lifted hyperbolic metric on $\widetilde{S}$ ), the hyperbolic geodesic $[\widetilde{\phi}(\lambda)]$ in $\widetilde{\mathfrak{M}}$ joining the endpoints of $\widetilde{\phi}(\lambda)$ lies outside the L-ball around $O \in \widetilde{\mathfrak{M}}$.

Proposition 4.4 follows from [39] Lemma 6.12, restated as Lemma 4.7 below. Theorem 4.3 follows immediately from Proposition 4.4 on applying Corollary 3.10 . The condition that $G_{\infty}$ be totally degenerate is introduced simply to avoid the annoyance of having to deal with geometrically finite ends.

Remark 4.5. We refer to the discussion in Section 3 for the equivalence of the condition as stated here with the condition on geodesics in the Cayley graph $\mathcal{G} \Gamma$.

Remark 4.6. Strictly speaking, since $S_{0}$ is $S$ with some Margulis tubes deleted, it cannot be identified with $S$. For a precise statement we need to work instead with welded split blocks in which the ends of the Margulis tubes deleted in the split blocks $B_{i}$ are reglued. Gluing the welded split blocks along their boundaries gives a welded model manifold $\mathfrak{M}_{\text {wel }}$ homeomorphic to $S \times \mathbb{R}$ or $S \times[0, \infty)$ according as $M$ is doubly or singly degenerate, see [39] $\S 4.3$ for details. For simplicity, we will ignore this distinction in the discussion below. 
The essence of Proposition 4.4 is contained in Lemma 4.7 below, whose proof occupies $\S 5$ and $\S 6$ of [39]. As in the bounded geometry case, the first step is to use $\lambda$ (or more precisely, the image $\widetilde{\phi}(\lambda)$ of $\lambda$ in $\widetilde{\mathfrak{M}}$ ) to construct a 'ladder' $\mathcal{L}_{\lambda}$ by 'flowing up' through the blocks of $\widetilde{\mathfrak{M}}$. By constructing a coarse retraction onto $\mathcal{L}_{\lambda}$, it is shown (39] Corollary 5.8) to be quasiconvex in the graph metric $d_{\text {graph }}$. The constants here are independent not only of $\lambda$, but also of the particular model $\mathfrak{M}$, depending in fact only on the topological type of the surface $S$. Starting from $\lambda$, this allows one to construct an 'admissible' path joining the endpoints of $\lambda$ which follows the levels $\mathcal{L}_{\lambda}$ and which, after a controlled sequence of alterations using a succession of different metrics, is ultimately modified into an electro-ambient quasi-geodesic in $\left(\widetilde{\mathfrak{M}}, d_{C H}\right)$ joining the endpoints of $\lambda$. Here $d_{C H}$ is the metric on $\widetilde{\mathfrak{M}}$ obtained by electrocuting the hyperbolic convex hulls $C H(\widetilde{K})$ of the extended split components $\widetilde{K}$ of $\widetilde{\mathfrak{M}}$. (Recall that an electro-ambient quasi-geodesic in $\left(\widetilde{\mathfrak{M}}, d_{C H}\right)$ is an electric quasi-geodesic whose intersection with each electrocuted component is geodesic in the hyperbolic (or model) metric, see Section 2.6.) This finally leads to the following key statement, which we once again formulate in the modified form appropriate to Corollary 3.10 .

Lemma 4.7 ([39] Lemma 6.12). Let $\mathfrak{M}$ be a model manifold of split geometry and without cusps for a fixed compact hyperbolizable surface $S$. Let $B_{0} \subset S_{0} \times[0,1]$ be a fixed base block and let $\phi: S \rightarrow \mathfrak{M}$ be an embedding which identifies $S$ with $S_{0} \times\{0\} \subset B_{0}$. Let $o \in S_{0} \subset \mathfrak{M}$ be a basepoint and fix a lift $O$ in the universal cover $\widetilde{S}=\mathbb{H}^{2}$. Let $\phi$ be the lift $\widetilde{\phi}: \widetilde{S} \rightarrow \widetilde{\mathfrak{M}}=\mathbb{H}^{3}$ so that $\widetilde{\phi}(O)=O$ as in Proposition 4.4.

Then for any $L>0$, there exists $f(L)>0$, such that for any geodesic segment $\lambda$ in $\left(\widetilde{S}, d_{S}\right)$ lying outside $B_{\mathbb{H}^{2}}(O, f(L)) \subset \widetilde{S}$, there is an electro-ambient quasi-geodesic $\gamma$ in $\left(\widetilde{\mathfrak{M}}, d_{C H}\right)$ joining the endpoints of $\widetilde{\phi}(\lambda)$ which lies outside $B_{\mathbb{H}^{3}}(O, L) \subset \widetilde{\mathfrak{M}}$.

To get from Lemma 4.7 to the statement of Proposition 4.4 , we have now to only to show that one can replace electro-ambient quasi-geodesic $\gamma$ in $\left(\widetilde{\mathfrak{M}}, d_{C H}\right)$ by the hyperbolic geodesic $[i(\lambda)]$ joining the endpoints of $\lambda$. This can be done in virtue of Lemma 2.6, see also [39] Lemma 2.5 and the short argument in the proof of [39] Theorem 7.1.

4.2.3. Proof of Theorem A: preliminaries. We want to adapt the above discussion to the situation of Theorem A, namely a sequence of geometrically finite purely loxodromic groups $G_{n}$ converging strongly to a degenerate group $G_{\infty}$.

First, suppose that we have a single type preserving discrete faithful representation $\rho: \Gamma \rightarrow$ $G$ where $\Gamma$ is geometrically finite purely loxodromic group and $G$ is a totally degenerate Kleinian group with incompressible ends. As discussed in $\$ 4.7$ of [39], Theorem 4.1 extends to any 
simply degenerate end of any hyperbolic manifold with incompressible ends. Thus one can easily modify Lemma 4.7 to apply in this more general situation.

The essence of the proof of Theorem $A$ is therefore to understand the dependence of the constants involved on the manifold $M=\mathbb{H}^{3} / G$. The manifold, and hence the ladder $\mathcal{L}_{\lambda}$ (really one ladder for each end of $M$ ), may not have bounded geometry, since its construction depends on the whole of each infinite end of $M$. However as already noted, the Lipschitz constant for the coarse projection to $\mathcal{L}_{\lambda}$ in the graph metric $d_{\text {graph }}$ depends only on the topological type of the surfaces defining the ends and is thus uniform over any approximating sequence $\rho_{n}$.

Next, we need to look into the effect on the constants involved of the modifications needed to get from the $d_{g r a p h}$-electro-ambient geodesic joining the endpoints of $\widetilde{\phi}(\lambda)$ as in [39] Section 6.1 (called $\beta_{e}$ in [39]) to a $d_{C H}$-electro-ambient geodesic with the same endpoints as in Lemma 4.7 and hence to a hyperbolic geodesic as in Proposition 4.4. Suppose we have a bounded segment $\gamma$ of such an electro-ambient geodesic which passes through a given finite collection of split blocks. Close examination of the proof in [39] shows that the modifications made in the course of replacing $\gamma$ with the corresponding segment $\hat{\gamma}$ of the required hyperbolic geodesic, depend only on the geometry of the hyperbolic convex hulls of the split components traversed by $\gamma$. The number $N(\gamma)$ of convex hulls traversed is bounded uniformly in terms of the graph quasi-convexity constant. Thus the modifications made to $\gamma$, which control how much nearer $\hat{\gamma}$ approaches the basepoint than $\gamma$, depend only the geometry of $N(\gamma)$ blocks, where $N(\gamma)$ depends only on the topology of the ends of $M$.

This leads to an equivalent reformulation of Lemma 4.7, which is essentially the same as [39] Corollary 6.13 in the context of a general hyperbolic manifold $M$ without cusps. We begin with some more notation. Suppose $M$ has ends $E^{1}, \cdots, E^{r}$, and that each $E^{k}$ is homeomorphic to $S^{k} \times[0, \infty)$ for some closed hyperbolic surface $S^{k}$, and such that each end is simply degenerate. Let $\mathcal{K} \hookrightarrow M$ be a Scott core cutting off the ends $E^{k}$, so that the boundary components of $\mathcal{K}$ are the surfaces $S^{k} \times\{0\}, i=1, \ldots, r$. Theorem 4.3 asserts that each end $E=E^{k}$ of $M$ has split geometry and has a model made by consistently gluing split blocks $B_{i}=B_{i}^{k}, i \in \mathbb{N}$, so that $B_{i-1}$ is glued to $B_{i}$ along their common boundary split surface $S_{i}=S_{i}^{k}$. For $q \in \mathbb{N}$, let $\mathcal{B}(q)=\mathcal{K} \cup \bigcup_{k=0}^{r} \bigcup_{i=0}^{q} B_{i}^{k}$ be the manifold formed by gluing the core $\mathcal{K}$ to the first $q$ blocks in each end as above. Replacing models by actual ends, we may assume that $\mathcal{B}(q)$ is quasiisometrically embedded in $M$.

We also change our formulation so as to be in accordance with the criterion in terms of $\mathcal{G} \Gamma$, see the explanation in Section 3 . Given an isomorphism $\rho: \Gamma \rightarrow G$, we have an embedding $j: \mathcal{G} \Gamma \rightarrow \mathbb{H}^{3}, j(\gamma)=\rho(\gamma) \cdot O$ of the Cayley graph of $\Gamma$ into $\mathbb{H}^{3}$. Recall also that if $\lambda$ is any 


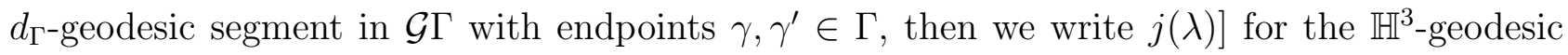
$\left[j(\gamma), j\left(\gamma^{\prime}\right)\right]$.

Remark 4.8. We also have the map $i: j_{\Gamma}(\mathcal{G} \Gamma) \rightarrow \mathbb{H}^{3}$ defined by $i j_{\Gamma}(\gamma)=j(\gamma)$, whose extension to $\Lambda_{\Gamma}$ is the $C T$-map $\hat{i}$. Note that $i$ is morally the same as the embedding $\widetilde{\phi}: \widetilde{S} \rightarrow \widetilde{\mathfrak{M}}$ of Proposition 4.4, and as long as $S$ is closed, the map $j_{\Gamma}$ is a quasi-isometry so that $i$ is also morally equivalent to $j$.

Proposition 4.9. Let $\Gamma$ be a geometrically finite convex cocompact Kleinian group and let $\rho: \Gamma \rightarrow G$ be a strictly type preserving isomorphism. Suppose that $M=\mathbb{H}^{3} / G$ is a hyperbolic 3-manifold without cusps, with $S$ cott core $\mathcal{K}$ and incompressible ends $E^{1}, \cdots, E^{r}$ as above. Then there exists $D \in \mathbb{N}$, depending only on the topology of the ends of $M$, with the following property. Suppose given $q \in \mathbb{N}$ and that the submanifold $\mathcal{B}(q)$ is defined as above. Then for all $L>0$ there exists $f(L)>0$, depending only on the geometry of the submanifold $\mathcal{B}(q+D)$, such that if $\lambda$ is any $d_{\Gamma}$-geodesic segment in $\mathcal{G} \Gamma$ which lies outside $B_{\Gamma}(1, f(L))$, then $[j(\lambda)] \cap \widetilde{\mathcal{B}(q)}$ lies outside $B(O, L)$, where $\widetilde{\mathcal{B}(q)}$ is the lift to $\widetilde{M}$ of $\mathcal{B}(q)$.

Thus the proposition asserts that, independent of the geometry of $M$ outside $\mathcal{B}(q+D)$, we can control $[j(\lambda)]$ inside $\widetilde{\mathcal{B}(q)}$ with constants which depend only on the first $q+D$ blocks in each end, where $D$ is a universal constant which depends only on the topological types of the ends.

We immediately deduce the following Corollary, which will be used in the proof of Theo$\operatorname{rem} \mathrm{A}$.

Corollary 4.10. Let $M, \mathcal{K}, D$ be as in Proposition 4.9. Let $q \in \mathbb{N}$. Then there exists $f: \mathbb{N} \rightarrow \mathbb{N}$ with the following property. Suppose that $V$ is any hyperbolic manifold such that there is a biLipschitz embedding $\beta: \mathcal{B}(q+D) \rightarrow V$, and such that the ends of $V \backslash \beta(\mathcal{B}(q+D))$ have split geometry and correspond bijectively and homeomorphically to the ends of $M \backslash \mathcal{B}(q+D)$. Let $\tilde{\beta}: \widetilde{\mathcal{K}} \rightarrow \widetilde{V}$ be the lift of $\beta$ to the universal covers. Suppose that $L>0$ and that $\lambda$ is any $d_{\Gamma}$-geodesic segment in $\mathcal{G} \Gamma$ which lies outside $B_{\Gamma}(1, f(L))$. Then $[\widetilde{\beta}(j(\lambda))] \cap \widetilde{\beta}(\widetilde{\mathcal{B}}(q))$ lies outside $B(\beta(O), L) \subset \widetilde{V}$, where $[\tilde{\beta}(j(\lambda))]$ denotes the geodesic whose endpoints are the images under $\tilde{\beta} \circ j$ of the endpoints of $\lambda$.

4.2.4. Proof of Theorem A: conclusion. We have a geometrically finite Kleinian group $\Gamma$ without parabolics, which does not split as a free product, together with a sequence of strictly type preserving isomorphisms $\rho_{n}: \Gamma \rightarrow G_{n}$ which converge strongly to a purely loxodromic Kleinian group $G_{\infty}=\rho_{\infty}(\Gamma)$. Our aim is to use the criterion of Corollary 3.10 to show that the corresponding sequence of $C T$-maps $\hat{i}_{n}: \Lambda_{\Gamma} \rightarrow \Lambda_{G_{n}}$ converges uniformly to $\hat{i}_{\infty}: \Lambda_{\Gamma} \rightarrow \Lambda_{G_{\infty}}$. 
Since the representations $\rho_{n}$ converge strongly to $G_{\infty}$, by Proposition 2.2 we may as well assume that we have a compact core $K$ of $N=\mathbb{H}^{3} / \Gamma$ together with embeddings $\phi_{n}: K \hookrightarrow$ $M_{n}=\mathbb{H}^{3} / G_{n}, n \in \mathbb{N} \cup \infty$, such that $K_{n}=\phi_{n}(K)$ is a Scott core of $M_{n}$ and $\mathcal{K}=\phi_{\infty}(K)$ is a Scott core of $M_{\infty}$. We may also choose the lift $O$ of the basepoint $o \in K$ and lifts $\widetilde{\phi}_{n}$ of $\phi_{n}$ so that for all $n, \tilde{\phi}_{n}(O)$ lies in a uniformly bounded neighborhood of $O \in \mathbb{H}^{3}$.

We need to show that there exists a function $f_{1}: \mathbb{N} \rightarrow \mathbb{N}$ such that whenever $\lambda$ is a $d_{\Gamma^{-}}$ geodesic segment lying outside $B_{\Gamma}\left(1 ; f_{1}(L)\right)$ in $\mathcal{G} \Gamma$, the $\mathbb{H}^{3}$-geodesic $\left[j_{n}(\lambda)\right]$ lies outside $B_{\mathbb{H}}(O ; L)$ for all $n \in \mathbb{N}$, where as usual $j_{n}: \mathcal{G} \Gamma \rightarrow \mathbb{H}^{3}, j_{n}(\gamma)=\rho_{n}(\gamma) \cdot O$.

As in 4.2 .3 above, let $E^{i}, i=1, \ldots, r$ be the ends of $M=M_{\infty}$. Given $L \in \mathbb{N}$, choose compact submanifolds $E_{1}^{i} \subset E^{i}$ homeomorphic to $S^{i} \times[0,1]$ such that $d_{M}\left(\left(E^{i} \backslash E_{1}^{i}\right), \mathcal{K}\right) \geq 2 L$, where $d_{M}$ denotes the metric induced by shortest paths in $M$. Identifying $M$ with the model $\mathfrak{M}$, pick $q \in \mathbb{N}$ so that $E_{1}^{i}$ is contained in the union of the first $q$ blocks of $E^{i}$ for each $i=1, \ldots, r$. Then with the notation of Proposition 4.9, we have $d_{M}(M \backslash \mathcal{B}(q), \mathcal{K}) \geq 2 L$.

By strong convergence, there exists $n_{0}=n_{0}(L) \in \mathbb{N}$ such that for all $n \geq n_{0}$, there exists a 2-bi-Lipschitz embedding $\psi_{n}: \mathcal{B}(q+D) \rightarrow M_{n}$, and such that $\psi_{n}(\mathcal{B}(q+D)) \supset K_{n}=\psi_{n}(\mathcal{K})$ cuts off the ends of $M_{n}$, see Lemma 2.1. Let $o \in \mathcal{K}$ be a base-point which lifts to $O \in \mathbb{H}^{3}$. Up to adjusting by a bounded distance, we may assume that $\psi_{n}$ lifts to a map $\tilde{\psi}_{n}$ with $\tilde{\psi}_{n}(O)=O$, and that $\tilde{\psi}_{n}(O)$ projects to the base-point $o_{n} \in M_{n}$ and $\psi_{n}(o)=o_{n}$.

Now apply Corollary 4.10 with $M=M_{\infty}$ and the integer $q$, and with $V=M_{n}$ and $\beta=\psi_{n}$, to see there exists a function $f: \mathbb{N} \rightarrow \mathbb{N}$, independent of $n$, with the following property. Let $\lambda$ be a $d_{\Gamma}$-geodesic segment in $\mathcal{G} \Gamma$ lying outside $B_{\Gamma}(1, f(2 L))$. Since $\left(\psi_{n}\right)_{*} \circ \rho_{\infty}=\rho_{n}$ we have $\widetilde{\psi}_{n} \circ j_{\infty}=j_{n}$. Let $\left[j_{n}(\lambda)\right]$ be the $\mathbb{H}^{3}$-geodesic segment with the same endpoints as $j_{n}(\lambda)$. Then by Corollary $4.10,\left[j_{n}(\lambda)\right] \cap \widetilde{\psi}_{n}(\widetilde{\mathcal{B}(q)})$ lies outside $B_{\mathbb{H}^{3}}(O, 2 L) \subset \mathbb{H}^{3}$, where $\widetilde{\mathcal{B}(q)} \subset \widetilde{M}_{\infty}$ is the lift to $\widetilde{M}_{\infty}=\mathbb{H}^{3}$ of $\mathcal{B}(q)$.

Let $\pi: \mathbb{H}^{3} \rightarrow M_{n}$ be the covering projection so that in particular $\pi(O)=o_{n}$. Since $\psi_{n}(\mathcal{B}(q))$ cuts off the ends of $M_{n}$, then since $\psi_{n}$ is 2-bi-Lipschitz, any point in $\pi\left(\left[j_{n}(\lambda)\right]\right)$ which lies outside $\psi_{n}(\mathcal{B}(q))$ must be at least distance $L$ to $o_{n}$. It follows that whenever $n \geq n_{0},\left[j_{n}(\lambda)\right]$ lies outside $B\left(\widetilde{\psi}_{n}(O), L\right) \subset \widetilde{M}_{n}=\mathbb{H}^{3}$ whenever $\lambda$ lies outside $B_{\Gamma}(1, f(L))$ in $\mathcal{G} \Gamma$.

It remains only to deal with $n<n_{0}$. By Corollary 3.10 , for each $n \in\left\{1, \ldots, n_{0}\right\}$, there exists $N_{n}=N_{n}(L) \in \mathbb{N}$, such that if $\lambda$ is a $d_{G^{-}}$geodesic outside $B_{\Gamma}\left(1, N_{n}(L)\right)$ in $\mathcal{G} \Gamma$, then $\left[j_{n}(\lambda)\right]$ lies outside $B(O, L) \subset \mathbb{H}^{3}$. Choosing $f_{1}(L)=\max \left\{f(L), N_{1}(L), \ldots, N_{n_{0}}(L)\right\}$ we have verified the criterion of Corollary 3.10. This completes the proof of Theorem A. 


\section{Algebraid limits And NON-COnVERGenCe of Limit POINTS}

In this section we prove Theorem $\mathrm{C}$, of which Theorem $\mathrm{B}$ is an immediate consequence. The sequence of groups $G_{n}$ in Theorem $\mathrm{C}$ is that described by Brock in [9], in which the convergence is algebraic but not strong. We begin with a brief description of the examples and Brock's bi-Lipschitz models for the manifolds involved.

5.1. Brock's Examples. The groups $G_{n}$ in Theorem C are a sequence of quasi-Fuchsian surface groups converging algebraically but not strongly to a partially degenerate geometrically infinite surface group $G_{\infty}$ with an accidental parabolic. The examples are also discussed briefly in [30].

The sequence $G_{n}$ is obtained as follows. Fix a closed hyperbolic surface $X=\mathbb{H}^{2} / \Gamma$. Let $\sigma$ be a simple closed geodesic which separates $X$ into two subsurfaces $R$ and $L$. Let $\alpha$ denote an automorphism of $X$ such that $\left.\alpha\right|_{L}$ is the identity and $\left.\alpha\right|_{R}=\chi$ is a pseudo-Anosov diffeomorphism of $R$ preserving the boundary $\sigma$. (For later reference, it is important to ensure that there is no Dehn twisting around $\sigma$ when $\chi$ is considered as the restriction of $\alpha$ to $\pi_{1}(R)$, see 5.3.1 below.) Let $G_{n}$ be the quasi-Fuchsian group given by the simultaneous uniformization of $\left(\alpha^{n}(X), X\right)$, so that $G_{n}=\rho_{n}(\Gamma)$ for suitably normalized $\rho_{n}: \Gamma \rightarrow S L(2, \mathbb{C})$ and $G_{0}$ is Fuchsian. This means that the regular set $\Omega_{n}$ of $G_{n}$ has two components $\Omega_{n}^{ \pm}$where the 'lower' component $\Omega_{n}^{-} / \Gamma$ is conformally equivalent to $X$ and the 'upper' component $\Omega_{n}^{+} / \Gamma$ is equivalent to $\alpha^{n}(X)$. The algebraic limit $G_{\infty}$ of the groups $G_{n}$ is a partially degenerate geometrically infinite surface group, while with suitable choice of basepoint, the geometric limit of the manifolds $M_{n}=\mathbb{H}^{3} / G_{n}$ is homeomorphic to $X \times \mathbb{R} \backslash R \times\{0\}$. These assertions will be explained in more detail below. Since to fully understand our example, it is important to be clear about the notational conventions, we begin by setting these out. As far as possible, we follow Brock 9 .

5.1.1. Teichmüller space and the mapping class group. Let $S$ be an oriented hyperbolizable surface. The Teichmüller space Teich $(S)$ of $S$ parametrizes finite area hyperbolic structures on $S$ up to isotopy. Thus a point $X \in \operatorname{Teich}(S)$ is a hyperbolic surface $X$ equipped with a homeomorphism $f: S \rightarrow X$ which marks $X$. The mapping class group $\operatorname{Mod}(S)$ acts on $\operatorname{Teich}(S)$ : if $\alpha \in \operatorname{Mod}(S)$ then $\phi(S, f)=\left(S, f \circ \alpha^{-1}\right)$. The map on surfaces induces an action on the space of representations $\rho: \pi_{1}\left(S, x_{0}\right) \rightarrow P S L_{2}(\mathbb{C})$ by $\alpha(\rho)=\rho \circ \alpha^{-1}$.

If $\zeta:[0,1] \rightarrow S$ is a path in $S$, we denote by $\alpha(\zeta)$ the path $\alpha \circ \zeta:[0,1] \rightarrow S$. Thus defining a hyperbolic surface $X$ in terms of the lengths $\ell_{X}(\gamma)$ of the simple closed curves $\gamma$ on $X$ and identifying $X$ with $S$ by taking $f=$ id, we can write

$$
\ell_{\alpha(X)}(\gamma)=\ell_{X}\left(\alpha^{-1}(\gamma)\right) .
$$


Hence the map $\alpha: X \rightarrow \alpha(X)$ is an isometry. The action on curves extends to an action on the space of measured laminations $\operatorname{ML}(S)$ on $S$ : for $\mu \in \operatorname{ML}(S)$ the lamination $\alpha(\mu)$ is defined by (geometric) intersection numbers:

$$
i(\gamma, \alpha(\mu))=i\left(\alpha^{-1}(\gamma), \mu\right)
$$

for all $\gamma \in \pi_{1}(S)$. Thus if $\alpha \in \operatorname{Mod}(S), \mu \in \operatorname{ML}(S)$ and $X \in \operatorname{Teich}(S)$ we have $\ell_{\alpha(X)}(\mu)=$ $\ell_{X}\left(\alpha^{-1}(\nu)\right)$ where $\ell_{X}(\mu)$ denotes the length of the lamination $\mu$ in the surface $X$.

5.1.2. Quasi-Fuchsian groups. A quasi-Fuchsian group $G$ is the image of a discrete faithful representation $\rho: \pi_{1}\left(S, x_{0}\right) \rightarrow P S L_{2}(\mathbb{C})$, whose domain of discontinuity has two simply connected components $\Omega^{ \pm}$. By convention we take $\Omega^{+}$to be the component whose orientation is the same as that of $S$. By Bers' simultaneous uniformisation theorem, a pair of points $X, Y \in \operatorname{Teich}(S)$ parametrize quasi-Fuchsian groups: if $G=G(X, Y)=\rho\left(\pi_{1}\left(S, x_{0}\right)\right)$ then $Q(X, Y)$ denotes the manifold for which $\Omega^{+} / G$ is conformally equivalent to $X$ and $\Omega^{-} / G$ is anti-conformally equivalent to $Y$. Strictly this only defines $G(X, Y)$ up to conjugation.

To mark $Q(X, Y)$ and fix $G(X, Y)$ we proceed as follows. Fix a base point $s_{0} \in S$. Let $Y$ be the point $(S, f) \in \operatorname{Teich}(S)$ and let $y_{0}=f\left(s_{0}\right)$. Fix a lift $\tilde{f}: \mathbb{H}^{2} \rightarrow \Omega^{-}$which descends to $f$, where we identify $\mathbb{H}^{2}$ with the universal cover of $S$ and where $O=O_{2} \in \mathbb{H}^{2}$ is a fixed basepoint which descends to $s_{0}$, and set $\tilde{y}_{0}=\tilde{f}\left(O_{2}\right)$. Now the convex hull $\mathcal{C}$ of $Q(X, Y)$ is bounded by two pleated surfaces $\partial \mathcal{C}^{ \pm}$which (when positively oriented, that is, so that $\partial \mathcal{C}^{+}$is oriented pointing out of $\mathcal{C}$ and $\partial \mathcal{C}^{-}$is oriented pointing into $\mathcal{C}$ ) are respectively uniformly bounded distance to $X, Y$ in Teich $(S)$. There is a natural retraction map $r$ from $\Omega^{-}$to the lift $\widetilde{\partial \mathcal{C}^{-}}$to $\mathbb{H}^{3}$ of $\partial \mathcal{C}^{-}$[16]; we arrange that $r\left(\tilde{y}_{0}\right)=O=O_{3} \in \mathbb{H}^{3}$. This gives a map $\tilde{f}:\left(\mathbb{H}^{2}, O_{2}\right) \rightarrow\left(\widetilde{\partial \mathcal{C}^{-}}, O_{3}\right)$ which descends to a map $S \rightarrow Q(X, Y)$ which sends $S$ to a pleated surface in $Q(X, Y)$ at uniformly bounded Teichmüller distance to $Y$. We use this marking to induce the representation $\rho: \pi_{1}\left(S, s_{0}\right) \rightarrow G(X, Y)$. We fix the basepoint $o \in Q=\mathbb{H}^{3} / G$ to be the projection of the point $O \in \mathbb{H}^{3}$.

5.1.3. Iteration of pseudo-Anosovs. For details on measured laminations and pseudo-Anosov maps, see [19, 41]. Here is a summary of what we need. Let $\chi \in \operatorname{Mod}(S)$ be pseudo-Anosov. Then $\chi$ has two fixed points in the space $\operatorname{PML}(S)$ of projective measured laminations on $S$ : the stable lamination $\lambda^{s}$ and an unstable lamination $\lambda^{u}$. (If necessary, we distinguish the underlying lamination $|\lambda|$ from its transverse measure $\lambda$.) This means there exists $c>1$ so that $\chi\left(\lambda^{s}\right)=\frac{1}{c} \lambda^{s}$ and $\chi\left(\lambda^{u}\right)=c \lambda^{u}$. From (2) this gives $i\left(\chi^{-1}(\gamma), \lambda^{u}\right)=i\left(\gamma, \chi\left(\lambda^{u}\right)\right)=c i\left(\gamma, \lambda^{u}\right)$. Thus $\lim _{n \rightarrow \infty} i\left(\chi^{n}(\gamma), \lambda^{u}\right) \rightarrow 0$ so that (since $\lambda^{u}$ is uniquely ergodic),

$$
\left[\chi^{n}(\gamma)\right] \rightarrow\left[\lambda^{u}\right] \text { in } \operatorname{PML}(\mathrm{S})
$$


where $[\mu]$ denotes the projective equivalence class of $\mu \in M L(S)$ in $\operatorname{PML}(S)$.

Let $X$ be a fixed surface in Teich $(S)$. The boundary leaves of laminations $\left|\lambda^{u}\right|,\left|\lambda^{s}\right|$ decompose $X$ into a collection of rectangles in each of which we have a metric $\sqrt{\left(\lambda^{u}\right)^{2}+\left(\lambda^{s}\right)^{2}}$ (or more simply the equivalent metric $\lambda^{u}+\lambda^{s}$ ). Putting these together gives a metric quasiisometric to the hyperbolic metric on $X$. Then $\ell_{X}(\gamma) \sim i\left(\gamma, \lambda^{u}\right)+i\left(\gamma, \lambda^{s}\right)$ and for any arc $T$ we have $\ell_{X}(T) \sim i\left(T, \lambda^{u}\right)+i\left(T, \lambda^{s}\right)$, where $\sim$ denotes equality up to multiplicative bounded constants. In particular, if $T$ is an arc along an unstable leaf then $\ell_{X}(T) \sim i\left(T, \lambda^{s}\right)$ so that $\ell_{\chi(X)}(T) \sim i\left(\chi^{-1}(T), \lambda^{s}\right)=c^{-1} i\left(T, \lambda^{s}\right) \sim c^{-1} \ell_{X}(T)$. In other words, $\phi$ contracts along unstable leaves. Also observe that since

$$
\ell_{\chi^{n}(X)}(\gamma)=\ell_{X}\left(\chi^{-n}(\gamma)\right) \sim i\left(\gamma, \chi^{n} \lambda^{s}\right)+i\left(\gamma, \chi^{n} \lambda^{u}\right)=c^{n} i\left(\gamma, \lambda^{u}\right)+c^{-n} i\left(\gamma, \lambda^{s}\right)
$$

it follows by taking ratios of lengths that $\chi^{n}(X) \rightarrow\left[\lambda^{u}\right]$ in $P M L(S)$, viewed as the Thurston compactification of Teich $(S)$.

5.1.4. The algebraic limit for iteration of pseudo-Anosov maps. Given a surface $S$ and $\alpha \in$ $\operatorname{Mod}(S)$, the mapping torus of $(S, \alpha)$ is the manifold $T_{\alpha}=S \times[0,1] / \sim$ where $\sim$ is the equivalence relation $(x, 0) \sim(\alpha(x), 1)$. Let $N_{\alpha}=S \times \mathbb{R}$ be the cyclic cover of $T_{\alpha}$, corresponding to the subgroup $\pi_{1}(S)$. The manifold $N_{\alpha}$ is naturally oriented by the orientation of $S$.

If $\chi \in \operatorname{Mod}(S)$ is pseudo-Anosov, then Thurston showed that $T_{\chi}$ and hence $N_{\chi}$ has a hyperbolic structure [41, 26]. Pick $(\Sigma, f) \in$ Teich $(S)$ and consider the manifold $M_{\chi}$ corresponding to the algebraic limit of the quasi-Fuchsian groups $G\left(\chi^{n}(\Sigma), \Sigma\right)$, where $Q\left(\chi^{n}(\Sigma), \Sigma\right)$ is marked as described above. McMullen [26] Theorem 3.11 shows that the limit manifold $M_{\chi}$ has one positive degenerate end $E$ which is asymptotically isometric to the positive end of $N_{\chi}$. (Note that the positive end of $N_{\chi}$ is, up to complex conjugation, the negative end of $N_{\chi^{-1}}$, see [26] Proposition 3.10.)

The end $E=E(\Sigma, \chi)$ consists of successive sheets which are mapped one to the next by $\chi$. More precisely, we have a sequence of pleated surfaces $h_{j}: S \rightarrow E$ exiting $E$ such that the $j^{t h}$ level surface is marked by the map $f \circ \chi^{-n}: S \rightarrow M_{\chi}$. In particular $h_{0}: S \rightarrow \Sigma \times\{0\}$ is the map $h_{0}(x)=\left(f^{-1}(x), 0\right)$ for $x \in \Sigma$; loosely, $h_{0}$ identifies $S$ with $\Sigma_{0}=\Sigma \times\{0\}$. Up to quasi-isometry, $E$ is modelled by $\Sigma \times[0, \infty)$ with the image of the $j^{\text {th }}$ level pleated surface $\Sigma_{j}$ identified with $\Sigma \times\{j\}$. The hyperbolic structure of $\Sigma_{j}$ is the point $\chi^{n}(\Sigma) \in \operatorname{Teich}(S)$. Now put a metric on $\Sigma \times[0,1]$ which smoothly interpolates between $\Sigma_{0}$ and $\Sigma_{1}$ and then transport this metric to $\Sigma \times[i, i+1]$ using the isometry $\chi^{i}$. This gives a uniformly bi-Lipschitz homeomorphism from $E$ to the model manifold $\Sigma \times[0, \infty)$. The model is marked by the map $h_{0} \circ f$ which sends a base-point $s_{0} \in S$ to a base-point $o=\left(f\left(s_{0}\right), 0\right) \in \Sigma_{0}$ 
The convex cores of the approximating manifolds $Q\left(\chi^{n}(\Sigma), \Sigma\right)$ are equally modelled by $\Sigma \times[0, n]$ with the restriction of the above metric. The above marking is, up to a uniformly bounded discrepancy, the same as the one described in 5.1.2, and hence determines the limit representation $\rho: \pi_{1}\left(S, s_{0}\right) \rightarrow \pi_{1}\left(M_{\chi}, o\right)$.

Lemma 5.1 ([9] Lemma 4.4). The ending lamination of the end $E$ of $M_{\chi}$ is the unstable lamination $\lambda^{u}$ of $\chi$.

Proof. This is proved in [9]. Here is a variant which will serve as a check we have the correct conventions. Think of the model $E=\Sigma \times[0, \infty)$ as quasi-isometrically embedded in $M_{\chi}$. Let $s_{0}$ be the base-point in $S$. As above, with $(\Sigma, f) \in \operatorname{Teich}(S)$ we have base-point $o=\left(f\left(s_{0}\right), 0\right) \in$ $\Sigma_{0} \subset M_{\chi}$. A loop $\gamma \in \pi_{1}\left(S, s_{0}\right)$ defines a path $(f \circ \gamma, 0) \subset \Sigma_{0}$ and hence a homotopy class $[\rho(\gamma)] \in \pi_{1}\left(M_{\chi}, o\right)$. Now consider the path $\chi^{n}(\gamma)=\chi^{n} \circ \gamma \in \pi_{1}(S)$. To find the approximate length of the geodesic in the class $\left[\rho\left(\chi^{n}(\gamma)\right)\right]$ in $M_{\chi}$, let $\tau_{n}$ be the path $t \mapsto\left(f\left(s_{0}\right), t\right), t \in[0, n] \subset$ $E$. Note that $\left(f \circ \chi^{n}(\gamma), 0\right)$ is homotopic in $M_{\chi}$ to the loop $\tau_{n}\left(f \circ \chi^{n}(\gamma), n\right) \tau_{n}^{-1}$ and hence freely homotopic to the path $\left(\chi^{n}(\gamma), n\right) \subset \Sigma_{n}$.

Now by (1), $\ell_{\Sigma_{n}}\left(\chi^{n}(\gamma)\right)=\ell_{\chi^{n}(\Sigma)}\left(\chi^{n}(\gamma)\right)=\ell_{\Sigma}(\gamma)$. Thus the sequence of curves $\left(\chi^{n}(\gamma), n\right)$ on the pleated surfaces $\Sigma_{n}$ exit the positive end of $M_{\chi}$ and have uniformly bounded length. This means they converge to the ending lamination of $M_{\chi}$. On the other hand, by (3), $\left[\chi^{n}(\gamma)\right] \rightarrow\left[\lambda^{u}\right]$ in $P M L(\Sigma)$. Hence the ending lamination of $M_{\chi}$ is $\lambda^{u}$.

5.1.5. The algebraic limit for iteration of partially pseudo-Anosov maps. Now we turn to the case under consideration, in which $\alpha \in \operatorname{Mod} S$ is partially pseudo-Anosov as described in Section 5.1 above. Thus $S$ is now a closed surface separated into two components $R$ and $L$ by a simple closed curve $\sigma$ and $\alpha \in \operatorname{Mod}(S)$ is such that $\left.\alpha\right|_{L}$ is the identity and $\left.\alpha\right|_{R}=\chi$ is a pseudo-Anosov diffeomorphism of $R$ preserving $\sigma$.

Given $X \in \operatorname{Teich}(S)$ we set $G_{n}=G\left(\alpha^{n}(X), X\right)$ and $M_{n}=Q\left(\alpha^{n}(X), X\right)$, so that $M_{n}$ is the manifold such that $\Omega^{+} / G_{n}$ is conformally equivalent to $\alpha^{n}(X)$ while $\Omega^{-} / G_{n}$ is anti-conformally equivalent to $X$. In particular, $G_{0}$ is a Fuchsian group uniformizing $X$ and our sequence of representations are the maps $\rho_{n}: G_{0} \rightarrow G_{n}$.

Brock showed, 9] Theorem 5.4, that the representations $\rho_{n}$ converge algebraically to a representation $\rho_{\infty}: G_{0} \rightarrow G_{\infty}$ where $G_{\infty}$ is a geometrically infinite surface group with corresponding manifold $M_{\infty}$. The regular set $\Omega_{\infty}$ of $G_{\infty}$ has one $G_{\infty}$-invariant simply connected component $\Omega_{\infty}^{-}$such that $\Omega_{\infty}^{-} / G_{\infty}$ is conformally equivalent to $X$. The positive end (corresponding to $\Omega^{+}$) has degenerated: if $g_{\sigma} \in \pi_{1}(S)$ corresponds to the separating curve $\sigma$, then $\rho_{\infty}\left(g_{\sigma}\right)$ is an accidental parabolic and $\Omega_{\infty}^{+}$collapses to a countable collection of simply connected components $\Omega^{+, i}$ whose stabilisers are each conjugate to $\rho_{\infty}\left(\pi_{1}(L)\right)$, so that $\Omega^{+, i} / G_{\infty}$ is 
a Riemann surface topologically equivalent to $\operatorname{Int} L$ for each $i$, with $\rho_{\infty}\left(g_{\sigma}\right)$ representing a loop encircling a puncture. Correspondingly, the upper end of $M_{\infty}$ is partially degenerate; the part corresponding to $L$ is geometrically finite while the part corresponding to $R$ is degenerate with ending lamination the unstable lamination of $\chi$.

Let $H$ be a horocyclic neighborhood of the cusp corresponding to $\rho_{\infty}\left(g_{\sigma}\right)$ in $M_{\infty}$. The assertion of [9] Theorem 5.4 is that the end of $M_{\infty} \backslash H$ cut off by the surface $R$ is asymptotically isomorphic to the end $E(\Sigma, \chi)$ described in the previous section, where $\Sigma$ is a hyperbolic surface with the same topology as Int $R$ but equipped with a complete hyperbolic structure so that the boundary $\sigma=\partial R$ is replaced by a cusp on $\Sigma$ and $\chi=\alpha_{\mid R}$.

5.1.6. Models of the approximating manifolds. Minsky [29] $\$ 6.5$ contains a description of the convex core of $M_{n}=Q\left(\alpha^{n}(X), X\right)$ in terms of a uniformly bi-Lipschitz model for its convex core $\mathcal{C}_{n}$. Fix $X \in \operatorname{Teich}(S)$ such that $\ell_{X}(\sigma)<\epsilon_{0}$ for some $\epsilon_{0}$ less than the Margulis constant. As above $\sigma$ separates $X$ into surfaces $R, L$; when needed we distinguish between the topological surfaces $R, L$ and the hyperbolic structures $X_{R}, X_{L}$ induced from $X$.

Also pick a complete hyperbolic surface $\Sigma$ with the same topology as $\operatorname{Int} R$ but so that the boundary $\sigma=\partial R$ is replaced by a cusp on $\Sigma$. Let $\Sigma^{c}$ denote $\Sigma$ with a small (open) neighborhood of the cusp removed so that the boundary curve, which we denote $\sigma^{c}$, has length $\epsilon_{0}$.

First we make a model $B_{n}$ for the part of $\mathcal{C}_{n}$ corresponding to $R$. Let $N=N_{\chi}$ be the hyperbolic 3-manifold with fiber $\Sigma$ and monodromy $\chi$. Let $N_{n}$ denote the cyclic $n$-fold cover of $N$, i.e. the manifold whose fundamental group is the kernel of the homomorphism $\pi_{1}(N) \rightarrow$ $\pi_{1}\left(S^{1}\right)=\mathbb{Z} \rightarrow \mathbb{Z}_{n}$. Let $N_{n}^{c}$ denote $N_{n}$ with a small (open) neighborhood of the cusp removed so that the boundary curve of each fiber has length $\epsilon_{0}$. Let $\hat{N}_{n}^{c}$ be the manifold obtained by cutting $N_{n}^{c}$ open along a lift of some fiber and completing metrically to a manifold with boundary. Then just as described in Section 5.1.4, there is a uniformly bi-Lipschitz homeomorphism from $\hat{N}_{n}^{c}$ to a model manifold $B_{n}=\Sigma^{c} \times[0, n]$, in which the block $\Sigma^{c} \times[i, i+1]$ is isometric to $\Sigma^{c} \times[0,1]$ by a map homotopic to $\chi^{-i}$. In the model metric on $B_{n}$, the boundary loop $\sigma^{c} \times\{t\}$ has length $\epsilon_{0}$ for each $t \in[0,1]$ and the boundary cylinder $\sigma^{c} \times[0, n]$ has the obvious product of the Euclidean metrics on $\sigma^{c}$ and the interval $[0, n]$. (The discussion in [7] $\S 8$, especially Proposition 8.6 for the discussion of $\partial \Sigma$, explains the model for $N_{n}^{c}$ in a neighborhood of a puncture.)

The model of the part of $\mathcal{C}_{n}$ corresponding to $L$ is essentially the product metric on $C=$ $X_{L} \times[0,1]$. We modify the metric on $X_{L}$ slightly to ensure the boundary circles $\partial L \times\{t\}$ all have length $\epsilon_{0}$ and take the standard Euclidean metric of length one on the second factor. 
Now we can make model $K_{n}$ for the whole of $\mathcal{C}_{n}$. Glue the circle $\partial L \times\{0\} \subset C_{n}$ to the circle $\partial R \times\{0\}=\sigma^{c} \times\{0\} \subset B_{n}$, and likewise glue the circle $\partial L \times\{1\}$ to the circle $\sigma^{c} \times\{n\}$. Let $K_{n}^{c}$ denote the resulting space. Let $\eta$ denote a circle of length $(n+1)$ obtained by moving in the direction of the second factor in $K_{n}^{c}$. Finally, let $K_{n}$ be the manifold (with boundary) obtained by (hyperbolic) Dehn filling $K_{n}^{c}$ with a Margulis tube $T_{n}$ with meridian $\eta$ and longitude $\sigma^{c}$, smoothing out at the boundary if needed. Figure 2 shows a 'cross-section' of $K_{n}$. Note that the manifolds $K_{n}$ have uniformly bounded geometry away from $T_{n}$.

The lower boundary of $K_{n}$, denoted $\partial K_{n}^{-}$, is obtained by gluing $\partial L \times\{0\}$ to $\partial R \times\{0\}$. Thus we have an obvious map $\phi_{n}: S \rightarrow \partial K_{n}^{-}$which we use to mark $K_{n}$. If $s_{0} \in \sigma$ is the base-point of $S$, we denote the image $\phi_{n}\left(s_{0}\right)=\left(s_{0}, 0\right) \in \partial L \times\{0\}$ by $o_{n}$. Lifting everything to universal covers, identifying $\widetilde{S}$ with $\mathbb{H}^{2}$, and thinking of $\widetilde{K}_{n} \subset \mathbb{H}^{3}$, we can arrange that $s_{0}$ lifts to $O \in \mathbb{H}^{2}$ and $o_{n}$ lifts to $O_{n}=O \in \mathbb{H}^{3}$.

The upper boundary of $K_{n}$, denoted $\partial K_{n}^{+}$, is obtained by gluing $\partial L \times\{1\}$ to $\partial R \times\{n\}$. This gives a second obvious embedding $\phi_{n}^{+}: S \rightarrow \partial K_{n}^{+}$and we write $o_{n}^{+}=\phi_{n}^{+}\left(s_{0}\right)$, with lift $O_{n}^{+} \in \mathbb{H}^{3}$. Occasionally we write $\phi_{n}^{-}$for $o_{n}, O_{n}^{-}$for $O_{n}=O$ and $\phi_{n}^{-}$for $\phi_{n}$ for clarity.

To see that the model manifolds $K_{n}$ are bi-Lipschitz equivalent to the convex cores $\mathcal{C}_{n}$, note that the marked surfaces $\partial K_{n}^{ \pm}$of $K_{n}$ are conformally a uniformly bounded Teichmüller distance from the surfaces $\alpha^{n}(X), X$ respectively, precisely as in the case of the manifolds $M_{n}=Q\left(\alpha^{n}(X), X\right)$. Thus the standard techniques used in the proof of the ending lamination theorem show that there are bi-Lipschitz homeomorphisms between $\mathcal{C}_{n}$ and $K_{n}$, with constants uniform in $n$. These are the models we will use.

5.1.7. The limit manifolds. In the algebraic limit, the tube $T_{n}$ becomes a rank one cusp, see also [30]. The lower boundary $\partial K_{n}^{-}$of $K_{n}$ stays fixed but the upper boundary $\partial K_{n}^{+}$develops into a partially degenerate end in which $L$ becomes a surface with a puncture. The part of the surface corresponding to $R$ becomes the degenerate end $E$ described in 5.1.6.

In the geometric limit, the distance from $o_{n}^{-} \in \partial K_{n}^{-}$to $o_{n}^{+} \in \partial K_{n}^{-}$stays bounded, because we can always travel through the bounded half $C=L \times[0,1]$. However to reach a point on a 'middle' layer $\Sigma^{c} \times\{n / 2\}$ of $B_{n}$ we have to travel ever further, either going directly 'up' through $B_{n}$ or through $L \times[0,1]$, crossing $\partial L \times\{1\}$, and then 'down' from $\Sigma^{c} \times\{n\}$ through $B_{n}$ to $\Sigma^{c} \times\{n / 2\}$. Thus in the geometric limit, $K_{n}$ converges to the manifold $S \times \mathbb{R} \backslash R \times\{0\}$ with two geometrically infinite ends, each asymptotically quasi-isometric to $E$. This is discussed in detail in [9, but is not important for us here.

5.2. Absence of Uniform Convergence. Since the sequence $G_{n}$ does not converge strongly, by Proposition 3.7, it must fail to satisfy UEP. In fact it is easy to exhibit a sequence of points 


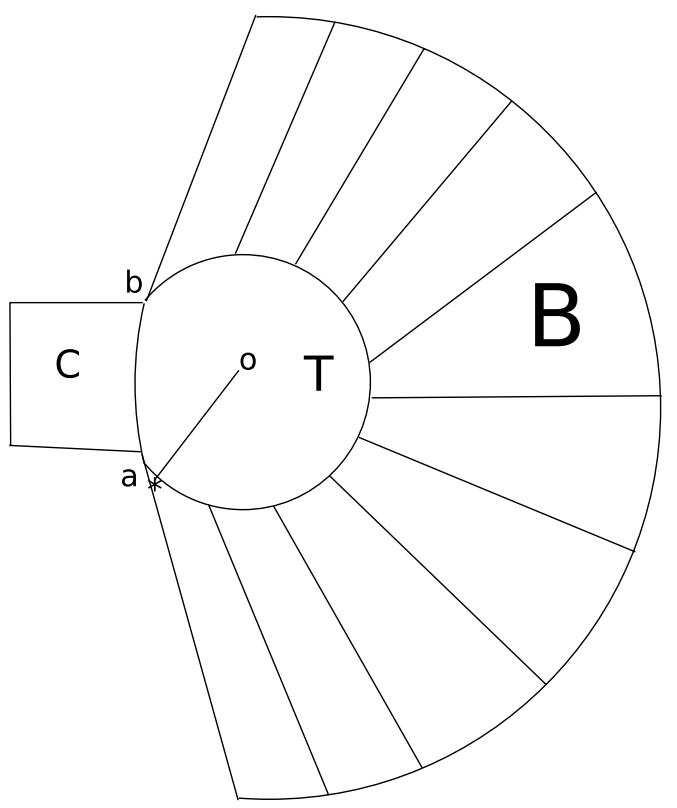

Figure 2. A schematic picture of $K$ built up of $B, C$ and $T$. The points $a, b$ are the base-points $\phi_{n}^{-}\left(s_{0}\right)=o_{n}^{-}$and $\phi_{n}^{+}\left(s_{0}\right)=o_{n}^{+}$respectively.

$g_{k} \in G_{0}$ such that $\left|g_{k}\right| \rightarrow \infty$ while $d\left(O, \rho_{n}\left(g_{k}\right) \cdot O\right) \leq c$ for all $n, k$ and some fixed $c>0$. (To see that this is equivalent to violating UEP, see [38] Lemma 5.2.)

The meridian curve $\eta$ round the boundary of the Margulis tube $T_{n}$ is split into two homotopic paths $\tau_{n}, v_{n}$ in $K_{n}$ by the points $o_{n}^{ \pm} \in \partial K_{n}^{ \pm}$. The path $\tau_{n}: t \mapsto\left(s_{0}, t\right), t \in[0, n]$ joins $o_{n}^{ \pm}$ going the 'long' way round $\partial T_{n}$ in $B_{n}$, while the path $v_{n}: t \mapsto\left(s_{0}, t\right), t \in[0,1]$ goes the 'short' way round in $C_{n}$.

Let $s \mapsto \gamma(s)$ be a based loop homotopic to a fixed generator of $\pi_{1}\left(S, s_{0}\right)$ and lying entirely on $R$. As in the proof of Lemma 5.1, the path $\gamma_{n}:[0,1] \rightarrow K_{n}, \gamma_{n}(s)=\left(\chi^{n} \gamma(s), n\right) \in \Sigma^{c} \times\{n\} \subset$ $\partial K_{n}^{+}$has the same length $\ell$ say as the path $s \mapsto \gamma_{0}(s)=(\gamma(s), 0) \in \Sigma^{c} \times\{0\} \subset \partial K_{n}^{-}$.

Now the loops $\tau_{n} \gamma_{n} \tau_{n}^{-1}$ and $v_{n} \gamma_{n} v_{n}^{-1}$ are homotopic in $K_{n}$, moreover from the above observation, $v_{n} \gamma_{n} v_{n}^{-1}$ has length $2+\ell$ in $K_{n}$. On the other hand in $K_{n}, \tau_{n} \gamma_{n} \tau_{n}^{-1}$ is homotopic to the loop $\chi^{n}\left(\gamma_{0}\right) \subset \Sigma^{c} \times\{0\} \subset \partial K_{n}^{-}$, where by $\chi^{n}\left(\gamma_{0}\right)$ we mean the path $s \mapsto\left(\chi^{n}(s), 0\right) \in \Sigma^{c} \times\{0\}$. By (4), the geodesic length of $\chi^{n}\left(\gamma_{0}\right)$ on $\Sigma^{c} \times\{0\}$ increases exponentially with $n$; hence by the usual comparison of word length and geodesic length on $\Sigma^{c} \times\{0\}$, if $g_{n} \in G_{0}$ represents the loop $\chi^{n}(\gamma) \in \pi_{1}\left(S, s_{0}\right)$ then $\left|g_{n}\right| \rightarrow \infty$ in $G_{0}$. Since $\rho_{n}$ is induced by the marking $\phi_{n}: s \mapsto(s, 0) \in K_{n}^{-}$, the loop $\chi^{n}\left(\gamma_{0}\right)$ is in the homotopy class of $\rho_{n}\left(g_{n}\right) \in \pi_{1}\left(K_{n} ; o_{n}^{-}\right)$.

Let $O$ be the lift to $\mathbb{H}^{3}$ of $o_{n}^{-} \in K_{n}$ as above. Lifting the paths $v_{n} \gamma_{n} v_{n}^{-1}$, we have found a sequence $g_{n} \in G_{0}$ for which $d_{G_{0}}\left(1, g_{n}\right) \rightarrow \infty$ but for which $d_{\mathbb{H}^{3}}\left(O, \rho_{n}\left(g_{n}\right) O\right)$ is uniformly bounded. As noted above, this violates UEP. 
5.3. Pointwise non-convergence. Let $\Gamma=G_{0}$ be the Fuchsian group for which $X=\mathbb{H}^{2} / \Gamma$. Recall that $\sigma$ corresponds to a loxodromic $g_{\sigma} \in \Gamma$ whose image under $\rho_{\infty}$ is parabolic. Let $\mathcal{P} \subset \Lambda_{\Gamma}$ denote the endpoints of axes which project to $\sigma$, equivalently, the set of images under $\Gamma$ of the fixed points of $g_{\sigma}$. The counter examples we seek for Theorem Cloccur in the case of $\xi \in \Lambda_{\Gamma}$ for which $\xi \notin \mathcal{P}$ but $\hat{i}_{\infty}(\xi)=\hat{i}_{\infty}(p)$ for some $p \in \mathcal{P}$. Precisely which points these are is given by the following theorem of Bowditch.

Theorem 5.2 ([7] Theorem 0.2). Let $\mathbb{H}^{2} / \Gamma$ be a punctured hyperbolic surface. Let $M=\mathbb{H}^{2} / G$ be a simply degenerate hyperbolic manifold corresponding to a faithful type preserving representation $\rho: \Gamma \rightarrow G$, and suppose that there is a lower bound to the length of all loxodromics in $M$. Suppose that $M$ has ending lamination $\lambda$ and let $\hat{i}: \Lambda_{\Gamma} \rightarrow \Lambda_{G}$ be the corresponding CT-map. Then $\hat{i}(\xi)=\hat{i}(\eta), \xi, \eta \in \Lambda_{\Gamma}$ if and only if $\xi$ and $\eta$ are either either ideal end-points of the same leaf of $\lambda$, or ideal boundary points of a complementary ideal polygon of $\lambda$.

This result was originally proved by Minsky [28] in the (bounded geometry) closed surface case. The condition on loxodromics means of course that the injectivity radius of $M$ is bounded below outside a horoball neighborhood of the punctures of $S$. This result has been extended to unbounded geometry and more general manifolds in [39], [33], [15].

5.3.1. The points of non-convergence. The points of non-convergence of the maps $\hat{i}_{n}$ will be the endpoints of lifts of unstable leaves which bound the crown domain of the unstable lamination $\left|\lambda^{u}\right|$ of $\chi$ in Int $R$. First, as mentioned above, we need to be careful about the precise meaning of saying that $\alpha_{\mid R}$ is pseudo-Anosov, so as to ensure that there is no Dehn twisting around $\sigma$ when we consider $\chi$ as the restriction of $\alpha$. We suppose given the hyperbolic surface $\Sigma$ as above and a pseudo-Anosov map $\chi: \Sigma^{c} \rightarrow \Sigma^{c}$ which pointwise fixes $\sigma^{c}$ and which is the identity in a horoball neighborhood of the cusp $\Sigma \backslash \Sigma^{c}$. Then $\chi$ induces an automorphism $\chi_{*}$ of $\pi_{1}\left(\Sigma^{c}, s_{0}\right)$, where we pick $s_{0} \in \partial \Sigma^{c}$. Now identify $R$ with $\Sigma^{c}$ and $\partial R=\sigma$ with $\partial \Sigma^{c}$. With this identification, we insist that $\left(\alpha_{*}\right)_{\mid \pi_{1}\left(R, s_{0}\right)}=\chi_{*}$.

Continuing with the identification of $R$ with $\Sigma^{c}$, note that the crown domain $F$ of the unstable lamination $\lambda^{u}$ of $\chi$ is an annulus with one boundary component $\sigma$ and the other consisting of finitely many alternating segments of stable and unstable leaves. By taking a suitable power of $\chi$ if necessary, we can assume that these leaves map to themselves under $\chi$.

Next, pick a lift $\tilde{F}$ of $F$ and a corresponding lift $\tilde{\chi}$ of $\chi$ which maps $\tilde{F}$ to itself and which is the identity on a particular lift $\tilde{\sigma}$ of $\sigma$. Let $\mu^{u}$ be the lift of one of the unstable leaves bounding the lift $\tilde{F}$ and let $\xi^{u}$ be one of its endpoints in $\Lambda_{\Gamma}$. From our assumption that $\left(\alpha_{*}\right)_{\mid \pi_{1}\left(R, s_{0}\right)}=\chi_{*}$, it follows that $\chi\left(\xi^{u}\right)=\xi^{u}$. (Without this assumption, we might have $\chi\left(\xi^{u}\right)=\rho\left(g_{\sigma}\right)^{k}\left(\xi^{u}\right)$ for some $k \in \mathbb{Z}$.) 
The non-convergence part of Theorem $\mathrm{C}$ is proved by

Proposition 5.3. Let $\xi^{u}$ be an endpoint of a boundary leaf of $\tilde{F}$, and let $p \in \mathcal{P}$ be an endpoint of the lift of $\sigma$ also bounding $\tilde{F}$. Then $\hat{i}_{\infty}\left(\xi^{u}\right)=\hat{i}_{\infty}(p)$, while no subsequence of the sequence $\hat{i}_{n}\left(\xi^{u}\right)$ limits on $\hat{i}_{\infty}(p)$.

Proof. The statement that $\hat{i}_{\infty}\left(\xi^{u}\right)=\hat{i}_{\infty}(p)$ follows from Theorem 5.2 since $\lambda^{u}$ is the ending lamination of $M_{\infty}$. The statement that no subsequence of the sequence $\hat{i}_{n}\left(\xi^{u}\right)$ limits on $\hat{i}_{\infty}(p)$ is Corollary 5.6 which we prove below.

To prove Corollary 5.6, we will construct, for each $n$, a quasi-geodesic in the lift $\widetilde{K}_{n}$ of $K_{n}$ which passes through the basepoint $O \in \widetilde{K_{n}}$, and with endpoints $\hat{i}_{n}\left(\xi^{u}\right)$ and $\hat{i}_{n}(p)$. These quasi-geodesics will be uniform in $n$ and the result will follow.

We want to consider $\xi^{u} \in \Lambda_{\Gamma}$ as a point in the limit set $\Lambda_{\Gamma}^{n}$ of the surface $\alpha^{n}(X)$. To do this, denote by $\mathbb{H}_{n}^{2}$ the universal cover of the surface $\alpha^{n}(X)$, with basepoint $\tilde{s}_{0}=\tilde{\chi}^{n}\left(\tilde{s}_{0}\right) \in \tilde{\sigma}$. The map $\tilde{\alpha}^{n}: \mathbb{H}^{2} \rightarrow \mathbb{H}_{n}^{2}$ extends to a homeomorphism $h_{n}: \Lambda_{\Gamma} \rightarrow \Lambda_{\Gamma}^{n}$. Since $\tilde{\alpha}^{n}\left(\mu^{u}\right)=\mu^{u}$, it follows that $h_{n}\left(\xi^{u}\right)=\xi^{u}$.

In $\mathbb{H}_{n}^{2}$, let $P_{n}$ be the foot of the perpendicular from $O=\tilde{s}_{0}$ to $\mu^{u}$ and consider the path $\beta_{n}$ which follows the perpendicular from $O$ to $P_{n}$ and then follows $\mu^{u}$ from $P_{n}$ to its endpoint $\xi^{u}$. The segment from $O$ to $P_{n}$ has length bounded independent of $n$ since outside the thin part of $X$, the diameter of the plaque $\tilde{F}$ is bounded. Hence $\beta_{n}$ is quasi-geodesic in $\mathbb{H}_{n}^{2}$.

The marking of $K_{n}$ is given by the embedding $\phi_{n}:\left(S, s_{0}\right) \rightarrow\left(\partial K_{n}^{-}, o_{n}^{-}\right)$which lifts to $\widetilde{\phi_{n}}:\left(\mathbb{H}^{2}, O\right) \rightarrow\left(\widetilde{K}_{n}, O\right)$. This extends to the map $\hat{i}_{n}: \Lambda_{\Gamma} \rightarrow \Lambda_{n}$, where $\Lambda_{n}$ is the limit set of $G_{n}$. On the other hand, the upper boundary $\partial K_{n}^{+}$of $K_{n}$ is marked by the map $\phi_{n}^{+}=\phi_{n} \circ \chi^{-n}$ whose lift $\widetilde{\phi_{n}^{+}}:\left(\mathbb{H}_{n}^{2}, O\right) \rightarrow\left(\widetilde{\partial K_{n}^{+}}, O_{n}^{+}\right)$extends to a map $q_{n}: \Lambda_{\Gamma}^{n} \rightarrow \Lambda_{n}$. Clearly, $q_{n} \circ h_{n}=\hat{i}_{n}$, so in particular, $q_{n}\left(\xi^{u}\right)=\hat{i}_{n}\left(\xi^{u}\right)$.

Lemma 5.4. The path $\widetilde{\phi_{n}^{+}}\left(\beta_{n}\right)$ from $O_{n}^{+}$to $\hat{i}_{n}\left(\xi^{u}\right)$ is quasi-geodesic in $\widetilde{K}_{n}$, with constants uniform in $n$.

Proof. The segment of $\widetilde{\phi_{n}^{+}}\left(\beta_{n}\right)$ from $O_{n}^{+}$to $\widetilde{\phi_{n}^{+}}(P)$ has uniformly bounded length, so it is sufficient to show that $\widetilde{\phi_{n}^{+}}\left(\mu_{n}\right)$ is uniformly quasi-geodesic in $\widetilde{K}_{n}$.

Suppose first that we were dealing with the case of a pseudo-Anosov map $\chi$ on a punctured hyperbolic surface $Y$, so that the stable and unstable laminations $\lambda^{s}, \lambda^{u}$ of $\chi$ fill up $Y$. Let $d x$ denote the transverse measure to $\lambda^{s}$ and $d y$ denote the transverse measure to $\lambda^{u}$, so that $d x$ measures length along unstable leaves and $d y$ measures length along stable leaves. As in 5.1.3, this defines a singular metric on $Y$ which for brevity we write as $d s^{2}=d x^{2}+d y^{2}$. 
Since $\chi$ expands along stable leaves, that is in the $y$-direction, the metric on $\chi^{n}(Y)$ is given by $d s^{2}=c^{-2 n} d x^{2}+c^{2 n} d y^{2}$. The same formula defines a singular metric on the universal cover $\mathbb{H}^{2}$.

Restricting the model end $E=Y \times[0, \infty)$ in 5.1 .3 to $E_{n}=Y \times[0, n]$ provides a model for the convex core of $Q\left(\chi^{n}(Y), Y\right)$. We have obvious maps which embed $Y$ and $\chi^{n}(Y)$ in $E_{n}$ as pleated surfaces $Y_{0}=Y \times\{0\}, Y_{n}=Y \times\{n\}$ respectively. Passing to universal covers, as in [28, see also [14], the metric in $\widetilde{E}_{n}$ is modelled by $d s^{2}=d t^{2}+c^{-2 t} d x^{2}+c^{2 t} d y^{2}$, where $t$ is the 'vertical' coordinate in the second factor. Thus the map which projects $\widetilde{E}_{n}$ 'vertically' upwards to $\widetilde{Y}_{n}=\tilde{Y} \times\{n\}$ is a contraction when restricted to $\mu^{u} \times[0, n]$, where as above $\mu^{u}$ is a boundary leaf of $\lambda^{u}$. Hence projecting from $\widetilde{E}_{n}$ to $\left(\mu^{u}, n\right)$ by first projecting 'horizontally' in the surface $\widetilde{Y}_{m}$ to $\left(\mu^{u}, m\right)$ and then 'vertically' to $\left(\mu^{u}, n\right)$ is a contraction, from which the result (that a leaf of the unstable lamination on the top surface $\widetilde{Y}_{n}$ is quasi-geodesic) follows by standard methods, see for example Bowditch [8] Lemma 4.2.

In the present case the model is somewhat more complicated because the automorphism $\alpha$ of the underlying surface $S$ is partially pseudo-Anosov and $K_{n}$ limits on a partially degenerate end of $M_{\infty}$. However we can apply the above argument working in the space in which we electrocute the left hand half $C_{n}=L \times[0, n]$, together with the Margulis tube $T_{n}$ around $\sigma_{n}$.

An equivalent proof can be constructed by modelling $\widetilde{K}_{n}$ as a tree of hyperbolic metric spaces as in [31].

Next, we modify the path $\widetilde{\phi_{n}^{+}}\left(\beta_{n}\right)$ of the previous lemma to a quasi-geodesic path from $O=O_{n}^{-}$with the same endpoint $\hat{i}_{n}\left(\xi^{u}\right) \in \Lambda_{n}$, by prefixing it with the path $v_{n}$ from $O_{n}^{-}$to $O_{n}^{+}$ which goes the 'short' way round $\partial T_{n}$ in $C_{n}$ as in section 5.2. Since $v_{n}$ has uniformly bounded length 1 , the resulting path $\delta_{n}$ is a $\widetilde{K}_{n^{-}}$quasi-geodesic from $O$ to $\hat{i}_{n}\left(\xi^{u}\right)$. It follows that the geodesic ray from $O$ to $\hat{i}_{n}\left(\xi^{u}\right)$ either lies completely outside $T_{n}$, or enters $T_{n}$ only to exit at a point $O^{\prime}$ a uniformly bounded distance from $O$.

Lemma 5.5. Let $p \in \mathcal{P}$ be as in the statement of Proposition 5.3. Let $\gamma_{n}$ be the hyperbolic ray from $O$ to $\hat{i}_{n}(p)$, and let $\delta_{n}$ be as above. Then the angle at $O$ between $\gamma_{n}$ and $\delta_{n}$ is uniformly bounded away from 0.

Proof. First consider first the limiting case in which $g_{\sigma}$ is parabolic. After normalizing and working in the upper half space model $\mathbb{H}^{3}$, we may assume we are in the following situation. Let $O \in \mathbb{H}^{3}$ be a fixed base point at Euclidean height 1 above the base plane. Suppose that $A \in \operatorname{Isom} \mathbb{H}^{3}$ is a parabolic fixing $\infty$. Suppose that $H$ is the height 1 horoball at $\infty$, so that $O \in \partial H$. Suppose that $\delta$ is a geodesic ray from $O$ which either lies completely outside $H$, or which enters $H$ and leaves it again at a point $O^{\prime}$ at distance at most $k$ from $O$. Let $\gamma$ be the 


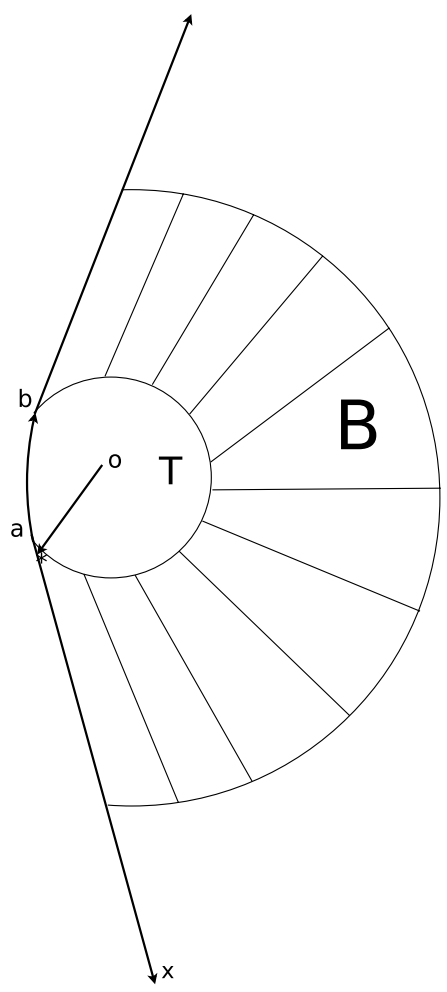

Figure 3. Geodesic realizations. The ray from $a=O_{n}^{-}$to $x$ (the image of a geodesic ray in $\widetilde{S}$ ) lies on the lower boundary $\partial \widetilde{K}_{n}^{-}$while its geodesic realization travels the short way round the (lifted) Margulis tube $\widetilde{T}$ to $b=O_{n}^{+}$and thence runs along the upper boundary $\partial \widetilde{K}_{n}^{+}$. The endpoints of the two rays coincide in $\partial \mathbb{H}^{3}$.

ray from $O$ to $\infty$. Then the angle $\alpha$ between $\delta$ and $\gamma$ at $O$ is bounded away from 0 ; precisely $2|\cot \alpha| \leq k^{\prime}$, where $k^{\prime}$ is the Euclidean bound on distance corresponding to the hyperbolic distance $k$.

Now we extend to the case of loxodromics of short translation length. Working in the upper half space model $\mathbb{H}^{3}$, let $A_{n} \in \operatorname{Isom} \mathbb{H}^{3}$ be a loxodromic fixing $\infty$. Suppose that the translation length $\ell\left(A_{n}\right) \rightarrow 0$ as $n \rightarrow \infty$. Let $T_{n}$ be a constant distance cone around Ax $A_{n}$, chosen so that the translation length of $A_{n}$ restricted to $\partial T_{n}$ is a fixed length $\epsilon_{0}$. Let $O \in \mathbb{H}^{3}$ be a fixed base point normalized to be at height 1 and assume that $O \in \partial T_{n}$. Let $a_{n}$ be the other end point of $\operatorname{Ax} A_{n}$. Let $\gamma_{n}$ be the geodesic ray from $O$ to $a_{n}$ and let $\delta_{n}$ be another geodesic ray from $O$ which either lies completely outside $T_{n}$, or which enters $T_{n}$ and leaves it again at a point $O^{\prime}$ at hyperbolic distance at most $k$ from $O$, where $k$ is bounded independent of $n$. We want to show that the angle between $\delta_{n}$ and $\gamma_{n}$ at $O$ is bounded away from 0 .

Let $\theta_{n}$ be the angle between the sides of the cone $\widetilde{T}_{n}$ and the horizontal. Since $\ell\left(A_{n}\right) \rightarrow 0$ while the translation length of $A_{n}$ restricted to $\partial \widetilde{T}_{n}$ is fixed, $\theta_{n} \rightarrow 0$ as $n \rightarrow \infty$. Now the angle 
between $\gamma_{n}$ and the vertical at $O$ is $2 \theta_{n}$. On the other hand, as is easy to compute, the angle between $\partial \widetilde{T}_{n}$ and $\delta_{n}$ at $O$ is uniformly bounded away from $\pi / 2$. Since $\partial \widetilde{T}_{n}$ is nearly horizontal, this proves the result.

Corollary 5.6. No subsequence of the sequence $\hat{i}_{n}\left(\xi^{u}\right)$ limits on the point $\hat{i}_{\infty}(p)$.

Proof. By Lemma 5.5, the visual angle subtended by $\hat{i}_{n}(p)$ and $\hat{i}_{n}\left(\xi^{u}\right)$ at $O$ is uniformly bounded below away from 0 . Since $p$ is the fixed point of an element of $\Gamma$, by algebraic convergence we have $\lim _{n \rightarrow \infty} \hat{i}_{n}(p)=\hat{i}_{\infty}(p)$ and the result follows.

Remark 5.7. In hindsight, Proposition 5.3 is perhaps not too unexpected as the paths $\tilde{\phi}_{n}^{+}\left(\beta_{n}\right)$ live on the top sheet of the approximating manifolds $\widetilde{K}_{n}$ and these converge to a ray whose limit does not lie in the limit set of the original surface subgroup $\pi_{1}(S)$.

5.4. Pointwise Convergence. We shall now establish that the $C T$-maps $\hat{i}_{n}: \Lambda_{\Gamma} \rightarrow G_{n}$ of the Brock examples converge pointwise for all points $\xi \in \Lambda_{\Gamma}$ other than those described in Proposition 5.3. We do this by applying the conditions $E P(\xi)$ (Embedding of Points) and $E P P(\xi)$ (Embedding of Pairs of Points) for pointwise convergence from [38]. These are essentially the criteria UEP and UEPP, relaxed so as to allow for dependence on the limit point $\xi$.

5.4.1. Convergence criteria. In [38] we described $E P(\xi)$ and $E P P(\xi)$ in relation to a sequence of elements $g_{i} \in \Gamma$ chosen so that $g_{i} \cdot O$ is a quasi-geodesic in $\mathcal{G} \Gamma$ and so that $g_{i} \cdot O \rightarrow \xi$ in the Euclidean metric on the ball model $\mathbb{B} \cup \partial \mathbb{B}$. It is easily seen that is equivalent to replace this with a criterion on the geodesic ray $[O, \xi)$ from $O$ to $\xi$ in the universal cover $\mathbb{H}^{2}$ of $X=\mathbb{H}^{2} / \Gamma$, where $X \in \operatorname{Teich}(S)$.

Definition 5.8. Let $\Gamma$ be a Fuchsian group such that $X=\mathbb{H}^{2} / \Gamma$ is a closed hyperbolic surface and let $\rho_{n}: \Gamma \rightarrow G_{n}$ be a sequence of isomorphisms to Kleinian groups $G_{n}$. Suppose given a sequence of $\left(\Gamma, G_{n}\right)$-equivariant embeddings $\tilde{\phi}_{n}:\left(\mathbb{H}^{2}, O\right) \rightarrow\left(\mathbb{H}^{3}, O\right)$ which induce basepoint preserving embeddings $\phi_{n}: X \rightarrow M_{n}$ with $\left(\phi_{n}\right)_{*}=\rho_{n}$. Let $\xi \in \Lambda_{\Gamma}$ and let $[O, \xi)$ be the geodesic ray in $\tilde{X}=\mathbb{H}^{2}$ as above.

(1) The pair $\left(\left(\rho_{n}\right), \xi\right)$ is said to satisfy $E P(\xi)$ if there exist functions $f_{\xi}: \mathbb{N} \rightarrow \mathbb{N}$ and $M_{\xi}: \mathbb{N} \rightarrow \mathbb{N}$, with $f_{\xi}(N) \rightarrow \infty$ as $N \rightarrow \infty$, such that for all $x \in[O, \xi)$ outside $B(O, N)$ in $\mathbb{H}^{2}, \widetilde{\phi}_{n}(x)$ is outside $B\left(O, f_{\xi}(N)\right)$ in $\mathbb{H}^{3}$, for all $n \geq M_{\xi}(N)$.

(2) The pair $\left(\left(\rho_{n}\right), \xi\right)$ satisfies $\operatorname{EPP}(\xi)$ if there exists a function $f_{\xi}^{\prime}(N): \mathbb{N} \rightarrow \mathbb{N}$ such that $f_{\xi}^{\prime}(N) \rightarrow \infty$ as $N \rightarrow \infty$, and such that for any subsegment $[x, y] \subset[O, \xi)$ lying outside $B(O ; N)$ in $\mathbb{H}^{2}$, the $\mathbb{H}^{3}$-geodesic $\left[\widetilde{\phi}_{n}(x), \widetilde{\phi}_{n}(x)\right.$ ] lies outside $B\left(O ; f_{\xi}^{\prime}(N)\right)$ in $\mathbb{H}^{3}$ for all $n \geq M_{\xi}(N)$, where $M_{\xi}$ is as in (1). 
Note that in these definitions, we do not assume that $M_{\xi}(N) \rightarrow \infty$ with $N$, in fact in the best situation, $M_{\xi}(N)=1$. We have:

Theorem 5.9 ([38] Theorem 7.3). Suppose that $\rho_{n}: \Gamma \rightarrow P S L_{2}(\mathbb{C})$ is a sequence of discrete faithful representations converging algebraically to $\rho_{\infty}: \Gamma \rightarrow P S L_{2}(\mathbb{C})$, and suppose the corresponding CT-maps $\hat{i}_{n}: \Lambda_{\Gamma} \rightarrow \Lambda_{G_{n}}$ exist, $n=1,2 \ldots, \infty$. Let $\xi \in \Lambda_{\Gamma}$. Then $\hat{i}_{n}(\xi) \rightarrow \hat{i}_{\infty}(\xi)$ as $n \rightarrow \infty$ if $\left(\left(\rho_{n}\right) ; \xi\right)$ satisfies $\operatorname{EPP}(\xi)$.

5.4.2. Verifying pointwise convergence. As above, we take $\Gamma=G_{0}$ and $\rho_{n}: G_{0} \rightarrow G_{n}$ to be the Brock examples as in Section 5.1.5. Sometimes it will be important to distinguish between the surface $S$ and the hyperbolic structure $X \in \operatorname{Teich}(S)$. Fixing such a structure $X$, we may take the dividing curve $\sigma$ to be geodesic on $X$. The restrictions $X_{R}, X_{L}$ of $X$ to $R, L$ are hyperbolic surfaces with geodesic boundary $\sigma$. The universal cover $\widetilde{S}$ of $S$ is identified with $\mathbb{H}^{2}$ using the lift of the structure $X$. Since $K_{n}$ is a quasi-isometric model for the convex core of $Q\left(\chi^{n}(X), X\right)$, we can identify the universal cover $\widetilde{K}_{n}$ of $K_{n}$ with a convex subset of $\mathbb{H}^{3}$.

The representations $\rho_{n}$ correspond to a sequence of embeddings $\widetilde{\phi}_{n}:\left(\mathbb{H}^{2}, O\right) \rightarrow\left(\mathbb{H}^{3}, O\right)$ which descend to the maps $\phi_{n}:\left(S, s_{0}\right) \rightarrow\left(X \times\{0\},\left(x_{0}, 0\right)\right) \subset K_{n}$. The map $\widetilde{\phi}_{n}$ extends to the $C T$-map $\hat{i}_{n}: \Lambda_{\Gamma} \rightarrow \Lambda_{n}$. Hence if $\xi \in \Lambda_{G_{0}}$, the ray $[O, \xi) \subset \mathbb{H}^{2}$ maps under $\widetilde{\phi}$ to a path $\widetilde{\phi}([O, \xi)) \subset \mathbb{H}^{3}$ joining $\widetilde{\phi}(O)=O$ to $\hat{i}_{n}(\xi) \in \Lambda_{n}$. We denote the $\mathbb{H}^{3}$-geodesic with these endpoints by $\left[O, \hat{i}_{n}(\xi)\right)$. We will prove convergence $\hat{i}_{n}(\xi) \rightarrow \hat{i}_{\infty}(\xi)$ by checking that $\left(\rho_{n}, \xi\right)$ satisfies the condition $\operatorname{EPP}(\xi)$.

5.4.3. Electric metrics. For $\xi \in \Lambda_{G_{0}}$, there are two possibilities for the geodesic ray $[O, \xi) \subset \widetilde{S}$ : either it is eventually contained in a fixed lift of $R$, or not. In the first case, translating by an appropriate element of $\Gamma=\pi_{1}(S)$ we may assume without loss of generality that the entire ray $[O, \xi)$ lies in a fixed lift of $R$.

Now consider the model manifold $K_{n}$ and let $D_{n}=B_{n} \cup T_{n} \subset K_{n}$. Since Margulis tubes are convex and since the tube $T_{n}$ separates $B_{n}$ from $C_{n}$, it follows that each lift $\widetilde{D_{n}}$ of $D_{n}$ is uniformly quasi-convex in $\widetilde{K_{n}}$. Hence $\left(\widetilde{K_{n}}, \mathcal{D}_{n}\right)$ satisfies the conditions of Lemma 2.6, where $\mathcal{D}_{n}$ is the collection of lifts $\widetilde{D_{n}}$ of $D_{n}$. Let $d_{e}^{n}$ denote the resulting electric metric on $\widetilde{K_{n}}$ with the collection $\mathcal{D}_{n}$ electrocuted.

Since the curve $\sigma$ along which we cut $X$ is geodesic, the lifts to $\mathbb{H}^{2}$ of $X_{R}$ are convex, moreover they are clearly uniformly separated. Let $d_{e}^{S}$ denote the induced electric metric on $\mathbb{H}^{2}$ with lifts of $X_{R}$ electrocuted. Clearly the ray $[O, \xi) \subset \mathbb{H}^{2}$ has infinite length in $d_{e}^{S}$ if and only if it is not eventually contained in a fixed lift of $R$.

Lemma 5.10. The map $\widetilde{\phi}_{n}:\left(\widetilde{S}, d_{e}^{S}\right) \rightarrow\left(\widetilde{K_{n}}, d_{e}^{n}\right)$ is a quasi-isometry with constants which are uniform in $n$. 
Proof. The map $\widetilde{\phi}_{n}$ is the lift to $\widetilde{S}$ of the map which sends $x \in L$ to $(x, 0) \in X_{L} \times[0,1]$ and $x \in R$ to $(x, 0) \in X_{R} \times[0, n]$. The lifts of the complement of $X_{L}$ are electrocuted in $\widetilde{S}$ and the lifts of the complement of $X_{L} \times[0,1]$ are electrocuted in $\widetilde{K_{n}}$. This result follows since $X_{L} \times[0,1]$ has vertical thickness 1 in $K_{n}$.

Corollary 5.11. The geodesic ray $\left[O, \hat{i}_{n}(\xi)\right) \subset \widetilde{K}_{n}$ has infinite length in the electric metric $d_{e}^{n}$ if and only if the ray $[O, \xi) \subset \widetilde{S}$ is not eventually contained in a fixed lift of $R$.

In the light of this corollary, the property of the ray $\left[O, \hat{i}_{n}(\xi)\right)$ having infinite $d_{e}^{n}$-length depends only on $\xi$. Thus we have two cases to consider depending on whether $[0, \xi)$ has finite or infinite length in the metric $d_{e}^{S}$. In both cases, to prove convergence $\hat{i}_{n}(\xi) \rightarrow \hat{i}_{\infty}(\xi)$, we will verify $\operatorname{EPP}(\xi)$.

5.4.4. Case 1: The length of $[0, \xi)$ in the electric metric $d_{e}^{S}$ is infinite. Let $\xi \in \Lambda_{\Gamma}$. First we prove $E P(\xi)$. Since $[O, \xi)$ has infinite $d_{e}^{S}$-length, no tail of $[O, \xi)$ is contained in a lift of $X_{R}$. Hence $[O, \xi)$ either crosses $X_{L}$ infinitely often, or has an infinite tail ending in a single lift of $X_{L}$. It follows that there exists a proper function $f_{\xi}: \mathbb{N} \rightarrow \mathbb{N}$ such that if $x \in[0, \xi)$ is at distance at least $N$ from $O$ in $\widetilde{S}$, then $d_{e}^{S}(O, x) \geq f_{\xi}(N)$.

By Lemma 5.10, the map $\widetilde{\phi}_{n}$ is a uniform quasi-isometry with respect to the respective electric metrics. Hence $d_{e}^{n}\left(O, \widetilde{\phi}_{n}(x)\right) \geq c^{\prime} f_{\xi}(N)$ for some constant $c^{\prime}>0$. Now any two lifts of $C_{n}=X_{L} \times[0,1]$ in $\widetilde{K}_{n}$ are separated by a constant $c>0$ which is also uniform in $n$. Hence $d_{\mathbb{H}^{3}}(u, v) \geq c d_{e}^{n}(u, v)$ for any points $u, v \in \widetilde{K}_{n}$. Absorbing the constants $c, c^{\prime}$ into the function $f_{\xi}$, we have shown that $d_{\mathbb{H}^{3}}\left(O, \widetilde{\phi}_{n}(x)\right) \geq f_{\xi}(N)$, which is just the statement $E P(\xi)$.

Now we prove $\operatorname{EPP}(\xi)$. Consider a segment $[a, b] \subset[O, \xi)$ such that $d(O, y) \geq N$ for all $y \in[a, b]$. From the above, $d_{\mathbb{H}^{3}}\left(O, \widetilde{\phi}_{n}(y)\right) \geq f_{\xi}(N)$ for all $y \in[a, b]$. In other words, $\widetilde{\phi}_{n}([a, b])$ lies outside $B\left(O, f_{\xi}(N)\right)$ in $\widetilde{K}_{n}$.

Now replace $[a, b]$ by the electro-ambient geodesic with the same endpoints. By Lemma 2.6, this is a bounded distance from $[a, b]$. It follows that $[a, b]$ is an electric quasi-geodesic in $\left(\widetilde{S}, d_{e}^{S}\right)$. Hence by Lemma 5.10, $\widetilde{\phi}_{n}([a, b])$ is an electric quasi-geodesic in $\left(\widetilde{K}_{n}, d_{e}^{n}\right)$ with constants which are uniform in $n$.

By Lemma 2.6 again, the $d_{e}^{n}$-electro-ambient geodesic obtained by replacing intersections of $\widetilde{\phi}_{n}([a, b])$ with the lifts $\widetilde{D}_{n}$ by hyperbolic geodesics in $\widetilde{D}_{n}$ with the same endpoints, is a uniform hyperbolic quasi-geodesic in $\widetilde{K}_{n}$ with the hyperbolic metric. Hence $\widetilde{\phi}_{n}([a, b])$ is a bounded distance away from the $\mathbb{H}^{3}$-geodesic with the same endpoints. This proves $\operatorname{EPP}(\xi)$. 
5.4.5. Case 2: The length of $[0, \xi)$ in the electric metric $d_{e}^{S}$ is finite. As before, let $\mathcal{P} \subset \Lambda_{G_{0}}$ denote the set of endpoints of lifts of the geodesic $\sigma$ and let $\mathcal{P}_{\infty} \subset \Lambda_{\infty}$ be the image of these points under $\hat{i}_{\infty}$. We will prove

Proposition 5.12. If the length of $[0, \xi)$ in the electric metric $d_{e}^{S}$ is finite and if $\hat{i}_{\infty}(\xi) \notin \mathcal{P}_{\infty}$, then $\lim _{N \rightarrow \infty} \hat{i}_{n}(\xi)=\hat{i}_{\infty}(\xi)$.

This will complete the proof of Theorem C. This proposition is the only point at which we use Theorem 4.2.

As above, $\Sigma$ is a surface with the same topology as $\operatorname{Int} R$ but equipped with a complete hyperbolic structure so that the boundary curve $\sigma$ is replaced by a puncture on $\Sigma$. We shall prove Proposition 5.12 by comparison with the behaviour of $C T$-maps for the sequence of quasi-Fuchsian groups $F_{n}$ uniformizing $\left(\chi^{n}(\Sigma), \Sigma\right)$ with corresponding manifolds $Q\left(\chi^{n}(\Sigma), \Sigma\right)$, so that in particular, $F_{0}$ is Fuchsian and $\mathbb{H}^{2} / F_{0}=\Sigma$. (Here $\chi$ is the same pseudo-Anosov map $\alpha_{\mid R}$ as above, extended as the identity map in a neighbourhood of the puncture on $\Sigma$.) As in Section 5.1.4, up to possibly passing to a subsequence, these groups limit on a simply degenerate group $F_{\infty}$ with corresponding manifold $M_{\chi}$ whose ending lamination is the unstable lamination of $\chi$. By [26] Theorem 3.12 the convergence is strong. Hence by Theorem 4.2 the CT-maps $\hat{k}_{n}: \Lambda_{F_{0}} \rightarrow \Lambda_{F_{n}}$ converge uniformly to the $C T$-map $\hat{k}_{\infty}: \Lambda_{F_{0}} \rightarrow \Lambda_{F_{\infty}}$. Thus the sequence of representations $\bar{\rho}_{n}: \pi_{1}(\Sigma) \rightarrow F_{n}$ satisfies $U E P P$. As above, we can model the convex cores of the manifolds $Q\left(\chi^{n}(\Sigma), \Sigma\right)$ by the restriction $E_{n}=\Sigma \times[0, n]$ of the end $E$ of $M_{\chi}$. These model manifolds $E_{n}$ are marked by the embedding $\psi_{n}: \Sigma \rightarrow \Sigma \times\{0\}$, which lifts to base points preserving embeddings $\left.\widetilde{\psi_{n}}:(\widetilde{\Sigma}, O) \rightarrow \widetilde{(\Sigma \times\{0\}}, O\right) \subset \widetilde{E}_{n}$.

To use the comparison between the representations $\bar{\rho}_{n}$ of $F_{0}=\pi_{1}(\Sigma)$ and $\rho_{n}$ of $G_{0}=\pi_{1}(S)$, we need to make definite the precise relationship between the limit sets $\Lambda_{F_{0}}$ and $\Lambda_{G_{0}}$. By definition the component $\Omega^{-}\left(G_{0}\right)$ of the regular set of $G_{0}$ projects to the Riemann surface $X$. Let $\widetilde{R}_{0}$ be a fixed component of the lift of $R$ to $\Omega^{-}\left(G_{0}\right)$, and let $J \subset G_{0}$ be its stabiliser, with corresponding limit set $\Lambda_{J} \subset \Lambda_{G_{0}}$. Then $\widetilde{R}_{0} / J$ can be identified with $X_{R}$ so that $J=\pi_{1}(R)$. Let $V$ be a bi-Lipschitz homeomorphism $X_{R} \rightarrow \Sigma^{c}$. Clearly $V$ induces an isomorphism $V_{*}: J \rightarrow F_{0}$ and a map $\widetilde{V}: \widetilde{R}_{0} \rightarrow \widetilde{\Sigma}^{c}$ which (see [38] Theorem 4.1) extends to a corresponding $C T$-map $\hat{i}_{V}: \Lambda_{J} \rightarrow \Lambda_{F_{0}}$.

Lemma 5.13. If $\hat{i}_{\infty}(\xi) \notin \mathcal{P}_{\infty}$, then $\left(\rho_{n}, \xi\right)$ satisfies $E P(\xi)$.

Proof. Translating by an appropriate element of $G_{0}$ we may assume without loss of generality that the geodesic ray $[0, \xi)$ is contained in $\widetilde{R}_{0}$ so that $\xi \in \Lambda_{J}$. Write $\bar{\xi}=\hat{i}_{V}(\xi)$. Since as above $\bar{\rho}_{n}$ satisfies $U E P P$, then certainly $\left(\bar{\rho}_{n}, \bar{\xi}\right)$ satisfies $\operatorname{EP}(\bar{\xi})$. Hence there is a strictly increasing function $f: \mathbb{N} \rightarrow \mathbb{N}$ such that if $x \in[O, \bar{\xi})$ and $d(O, x)>N$ then $d_{\mathbb{H}^{3}}\left(\widetilde{\psi}_{n}(x), O\right)>f(N)$. 
Now given $N \in \mathbb{N}$ and $\bar{x} \in[O, \bar{\xi})$, consider the $\mathbb{H}^{3}$-geodesic segment $\lambda=\left[O, \widetilde{\psi}_{n}(\bar{x})\right]$ from $O$ to $\widetilde{\psi}_{n}(\bar{x})$, and let $\mathcal{H}(\lambda)$ denote the collection of horoballs traversed by $\lambda$. Let $P_{N}(\bar{x}, n)$ be the total length of the geodesic segments in $\left[O, \widetilde{\psi}_{n}(\bar{x})\right] \cap \mathbb{H}^{3} \backslash \mathcal{H}(\lambda)$ and $Q_{N}(\bar{x}, n)=|\mathcal{H}(\lambda)|$ be the number of horoballs traversed by $\lambda$. We claim there exist a strictly increasing function $g: \mathbb{N} \rightarrow \mathbb{N}$ and $M_{N} \in \mathbb{N}$ such that

$$
P_{N}(\bar{x}, n)+Q_{N}(\bar{x}, n) \geq g(N) \text { for all } \bar{x} \in[O, \bar{\xi}), \bar{x} \notin B_{\mathbb{H}^{3}}(O, N) \text { and } n \geq M_{N} \text {. }
$$

If the claim is false, then there exists $K>0$ such that for all $N$, there exist $\bar{x}_{N} \in[O, \bar{\xi})$, $\bar{x}_{N} \notin B_{\mathbb{H}^{3}}(O, N)$ and arbitrarily large $n \in \mathbb{N}$ such that

$$
P_{N}\left(\bar{x}_{N}, n_{N}\right)+Q_{N}\left(\bar{x}_{N}, n_{N}\right) \leq K .
$$

Inductively, choose $n=n_{N}>n_{N-1}$. Then (6) implies in particular that there is a uniform bound to the number of horoballs traversed by the ray $\left[O, \widetilde{\psi}_{n_{N}}\left(\bar{x}_{N}\right)\right]$. By slightly adjusting constants, we can assume that each horoball is penetrated to a distance at least $a>0$ for some $a$. Now by hypothesis $d\left(O, \widetilde{\psi}_{n_{N}}\left(\bar{x}_{N}\right)\right) \geq f(N)$. Thus there can be no uniform upper bound to the distance travelled through each horoball, in other words, we can find a sequence $H_{N}$ of horoballs such that the length of the segment $\widetilde{\psi}_{n_{N}}\left(\bar{x}_{N}\right) \cap H_{N}$ tends to infinity with $N$. By choosing the first such horoball traversed, we can assume that $H_{N}$ intersects $B_{\mathbb{H}}(O, K)$. Passing to a subsequence if necessary, we may assume that all horoballs $H_{N}$ are based at the point $\hat{k}_{n}(\eta)$ for some fixed parabolic point $\eta \in \Lambda_{F_{0}}$. Thus we can find a sequence $y_{N} \in\left[O, \bar{x}_{N}\right]$ such that $\widetilde{\psi}_{n_{N}}\left(y_{N}\right) \in H_{N}$ and $d\left(O, \widetilde{\psi}_{n_{N}}\left(y_{N}\right)\right) \rightarrow \infty$. Since the rays $\widetilde{\psi}_{n_{N}}([O, \bar{\xi}))$ converge to $\widetilde{\psi}_{\infty}([O, \bar{\xi}))$ uniformly on compact subsets in $\mathbb{H}^{3}$ (by UEPP for the sequence $\bar{\rho}_{n}$ ), this means that $\left.\widetilde{\psi}_{n_{N}}\left(y_{N}\right)\right) \rightarrow \hat{j}_{\infty}(\eta)$. On the other hand, $\widetilde{\psi}_{n_{N}}\left(y_{N}\right)$ is arbitrarily close in the Euclidean metric on $\mathbb{B} \cup \hat{\mathbb{C}}$ to $\hat{k}_{n_{N}}(\eta)$ for large $N$. Hence $\hat{k}_{\infty}(\bar{\xi})=\hat{k}_{\infty}(\eta)$.

Since $\eta$ is a parabolic point in $\Lambda_{F_{0}}$, by Bowditch's Theorem 5.2 this means that either $\bar{\xi} \in V_{*}(\mathcal{P})$ or $\bar{\xi}$ is the endpoint of a leaf in the crown of the unstable lamination of $\chi$. Since $\hat{i}_{V}: \Lambda_{J} \rightarrow \Lambda_{F_{0}}$ is one-to-one except on $\mathcal{P}$, the same is true of $\xi$. Since by assumption $\xi \notin \mathcal{P}$, we deduce that $\xi$ is the end of a boundary leaf of the crown of $\chi$, which gives, using Theorem 5.2 again, $\hat{i}_{\infty}(\xi) \in \mathcal{P}_{\infty}$, contrary to hypothesis. This proves claim (5).

Now we will show that claim (5) implies that $\left(\rho_{n}, \xi\right)$ satisfies $E P(\xi)$. As above, let $D_{n}=$ $B_{n} \cup T_{n}$ and let $\widetilde{D}_{n}$ denote the lift of $D_{n}$ corresponding to $\widetilde{R}_{0}$ above, that is, whose stabiliser is $\rho_{n}(J)$. Let $\widetilde{B}_{n}$ be the corresponding lift of $B_{n}$. The map $V$ induces an obvious uniformly bi-Lipschitz map $V_{n}: B_{n}=X_{R} \times[0, n] \rightarrow E_{n}^{c}=\Sigma^{c} \times[0, n]$, where $E_{n}=\Sigma \times[0, n]$ is the model of the convex core of $Q\left(\chi^{n}(\Sigma), \Sigma\right)$ as in Section 5.1.6. Clearly $V_{n} \circ \phi_{n}=\psi_{n} \circ V$, while on the level of fundamental groups, $\left(V_{n}\right)_{*} \circ \rho_{n}=\bar{\rho}_{n} \circ V_{*}$ and $\left(V_{n}\right)_{*}, V_{*}$ are group isomorphisms. 
Since Margulis tubes are convex, it follows as in [20] that $\widetilde{D}_{n}$ satisfies the condition of Lemma 2.6 relative to the collection $\mathcal{T}_{n}$ of lifts of $T_{n}$ it contains, as does $\widetilde{E}_{n}$ relative to the set of horoballs $\mathcal{H}_{n}$ say. Let $\widetilde{D}_{n}^{e}, \widetilde{E}_{n}^{e}$ denote the corresponding electric spaces. Note that $V_{*}$ induces a bijective correspondence between $\mathcal{T}_{n}$ and $\mathcal{H}_{n}$.

To avoid having to define the extension of $\widetilde{V}_{n}$ to the whole of $\widetilde{D}_{n}^{e}$ we proceed as follows. Suppose that $\lambda$ is an electric quasi-geodesic in $\widetilde{D}_{n}^{e}$ with endpoints in $\widetilde{B}_{n}$. Replace $\lambda$ with a path $\hat{\lambda}$ which runs along the boundaries of the electrocuted sets in $\mathcal{T}_{n}$ as follows. Suppose some segment $\lambda^{\prime}$ of $\lambda$ enters and leaves some $T \in \mathcal{T}_{n}$ at points $a, b$ respectively. Replace $\lambda^{\prime}$ by the segment $(a,[0,1]) \cup(b,[0,1]) \subset \partial T \times[0,1]$ of electric length 2 . Since the sets in $\mathcal{T}$ are uniformly separated, the resulting path $\hat{\lambda}$ is still an electric quasi-geodesic. Now extend the definition of $\widetilde{V}_{n}$ to a map, still denoted $\widetilde{V}_{n}$, which sends $\partial T \times[0,1] \rightarrow \partial H \times[0,1]$ in the obvious way, where $H \in \mathcal{H}$ corresponds to $T \in \mathcal{T}$. Using the fact that $V_{n}$ is uniformly bi-Lipschitz, it is easy to see that $\widetilde{V}_{n}(\hat{\lambda})$ is an electric quasi-geodesic in $\widetilde{E}_{n}^{c}$, and that the number of electrocuted components traversed by $\hat{\lambda}$ and $\widetilde{V}_{n}(\hat{\lambda})$ is the same.

Now suppose $\xi \in \Lambda_{J}$ as in the statement of the Lemma. Since $\xi \notin \mathcal{P}$ the ray $[O, \xi)$ is contained in the convex hull of $\widetilde{X}_{R} \subset \widetilde{X}$. Note however that the path $\widetilde{V}([O, \xi))$ may not be a quasi-geodesic in $\widetilde{\Sigma}$ as it is contained in $\Sigma^{c}$ and thus may skirt round the boundaries of horoballs in $\Sigma$. We can nevertheless work with the ray $\widetilde{V}([O, \xi))$, which ends at the point $\hat{i}_{V}(\xi)=\bar{\xi}$, see for example [38] Theorem 4.1. (We obtain a quasi-geodesic ray from $\widetilde{V}([O, \xi)$ ) by replacing each segment which skirts a horoball with the corresponding geodesic joining the entry and exit points, see for example [38] especially Lemma A.5.)

Let $x \in[O, \xi)$ and let $\bar{x}=\widetilde{V}(x)$. We want to compare the ray $\left[O, \widetilde{\phi}_{n}(x)\right] \subset \widetilde{K}_{n}$ to the ray $\left[O, \widetilde{\psi}_{n}(\bar{x})\right] \subset \widetilde{E}_{n}$. Since $\widetilde{D}_{n}$ is quasi-convex in $\widetilde{K}_{n}$, we can after bounded adjustments assume that $\left[O, \widetilde{\phi}_{n}(x)\right] \subset \widetilde{D}_{n}$. Since $\widetilde{V}_{n} \widetilde{\phi}_{n}=\widetilde{\psi}_{n} \widetilde{V}$ we have $\widetilde{V}_{n}\left(\widetilde{\phi}_{n}(x)\right)=\widetilde{\psi}_{n}(\bar{x})$.

Replacing the electric geodesic $\lambda$ say from $O$ to $\widetilde{\phi}_{n}(x)$ in $\widetilde{D}_{n}^{e}$ by the corresponding electric quasi-geodesic $\hat{\lambda}$ as above, we see that $\widetilde{V}_{n}(\hat{\lambda})$ is a well-defined electric quasi-geodesic in $\widetilde{E}_{n}^{e}$ with endpoint $\widetilde{\psi}_{n}(\bar{x})$. Moreover $\widetilde{V}_{n}(\hat{\lambda})$ has length comparable to $\hat{\lambda}$. Since (5) effectively says that the length of $\widetilde{V}_{n}(\hat{\lambda})$ in the electric metric on $\widetilde{E}_{n}^{e}$ goes to infinity uniformly with $N$ independently of $n$, the same is true of $\hat{\lambda}$. This proves that $\left(\rho_{n}, \xi\right)$ satisfies $E P(\xi)$ and we are done.

Corollary 5.14. If $\hat{i}_{\infty}(\xi) \notin \mathcal{P}_{\infty}$, then $\left(\rho_{n}, \xi\right)$ satisfies $\operatorname{EPP}(\xi)$.

Proof. Continuing with the notation of Lemma 5.13, let $\lambda$ be a geodesic segment in $[O, \xi)$ outside $B_{\mathbb{H}^{2}}(O, N)$ and let $\bar{\lambda}=\widetilde{V}(\lambda)$. As in the previous lemma, note that $\bar{\lambda}$ may not be a geodesic as it is contained in $\Sigma^{c}$ and thus may skirt round the boundary of a horoball in $\Sigma$. This leads to an annoying technical issue in that it is convenient only to work with segments $\bar{\lambda}$ whose endpoints 
lie outside the lifts of the horoball $\Sigma \backslash \Sigma^{c}$. To fix this, note that in Definition 5.8 of condition $\operatorname{EPP}(\xi)$ for convergence, it is clearly enough to check the condition for an increasing sequence of values $N_{1}<N_{2}<\ldots$. Since $\widetilde{V}([O, \xi))$ does not terminate in the cusp, we may therefore restrict to those $N_{i}$ for which the first $x \in \widetilde{V}([O, \xi))$ outside $B\left(O, N_{i}\right)$ is outside a horoball. Thus given $\bar{\lambda}$ as above, by extending forwards and backwards along $\widetilde{V}([O, \xi))$ if necessary, we may assume that its initial and final points are outside horoballs in $\widetilde{\Sigma}$.

Now consider the geodesic $\left[\widetilde{\psi}_{n}(\bar{\lambda})\right]$ in $\widetilde{E}_{n}^{e}$ and let $\mu, \mu_{e a}$ be respectively the electric geodesic and the electro-ambient geodesic with the same endpoints, as in Section 2.6. By Lemma2.6, $\mu_{e a}$ is a bounded distance from $\left[\widetilde{\psi}_{n}(\bar{\lambda})\right]$. Using $E P P(\bar{\xi})$, we deduce that $\mu_{e a}$ is outside $B_{\mathbb{H}^{3}}(O, g(N)-$ $k$ ), for some uniform $k>0$. Then using the same method as in the previous lemma, it follows that any point on $\mu$ is outside some ball $B(O, h(N))$ in the electric metric on $\widetilde{E}_{n}^{e}$ for some function $h(N) \rightarrow \infty$ with $N$.

Now using the same trick as in the previous lemma, replace $\mu$ with the electric quasigeodesic $\hat{\mu}$ and apply the map $\widetilde{V}_{n}^{-1}$. We obtain an electric quasi-geodesic $\hat{\nu}=\widetilde{V}_{n}^{-1}(\hat{\mu})$ in $\widetilde{D}_{n}^{e}$ with the same endpoints as $\widetilde{\phi}_{n}(\lambda)$. Since $\widetilde{V}_{n}^{-1}$ is bi-Lipschitz with respect to electric metrics, for any point $Q \in \hat{\nu}$ we have $d_{e}(O, Q) \succ h(N)$, where we write $X \succ Y$ to mean there is a uniform constant $c>0$ such that $X>c Y$.

By Lemma 2.6 again, it will be enough to show that the electro-ambient quasi-geodesic obtained from $\hat{\nu}$ by replacing each segment which cuts through an equidistant tube $T \in \mathcal{T}_{n}$ with the hyperbolic geodesic with the same endpoints, is outside some ball $B_{\mathbb{H}}(O, f(N))$ for some function $f(N) \rightarrow \infty$ with $N$.

Suppose that $A$ and $B$ are the entry and exit points of $\hat{\nu}$ to some $T \in \mathcal{T}_{n}$. Suppose that the hyperbolic geodesic from to $O$ to $A$ first meets $T$ at a point $\bar{A}$. Since $d_{e}(O, A) \succ h(N)$, it follows that $d_{\mathbb{H}}(O, \bar{A}) \succ h(N)$. We deduce from Lemma 5.15 below that $T$ is entirely outside $B(O, R)$ for some $R \succ h(N)$. In particular the hyperbolic geodesic segment $[A, B]$ is outside $B(O, R)$ and the result follows.

Lemma 5.15. Suppose that $T$ is an equidistant tube in $\mathbb{H}^{3}$, that is, the set of points equidistant from a geodesic axis in $\mathbb{H}^{3}$, and that $T$ has radius at least $r$ for some uniformly large $r$. Suppose that $A \in \partial T$ is outside $B(O, R)$, where $O \in \mathbb{H}^{3}$ is a fixed base-point. Then the entire tube $T$ is outside $B\left(O, R^{\prime}\right)$ for some $R^{\prime} \succ R$.

Proof. Let $P$ be the point on $T$ nearest to $O$ in the hyperbolic metric and let $P^{\prime}, A^{\prime}$ be the feet of the perpendiculars from $P, A$ to the axis of $T$. We claim that if $d(A, P)>c>1$, then the angle $\theta$ between the geodesics $[A, P]$ and $\left[A, A^{\prime}\right]$ is uniformly bounded away from $\pi / 2$. If $d\left(A^{\prime}, P^{\prime}\right)>1$, this is easy since $[A, P]$ roughly tracks the quasi-geodesic $\left[A, A^{\prime}\right] \cup\left[A^{\prime}, P^{\prime}\right] \cup\left[P^{\prime}, P\right]$. 
If $d\left(A^{\prime}, P^{\prime}\right) \leq 1$ let $Q, Q^{\prime}$ be respectively the feet of the perpendiculars from $A^{\prime}, P^{\prime}$ to the geodesic $[A, P]$, so that $\left|Q Q^{\prime}\right|<1$. Since $\cos \theta=\tanh |A Q| / \tanh \left|A A^{\prime}\right|$ and $\left|A A^{\prime}\right| \geq r$, it follows that $\cos \theta$ is bounded away from 0 unless $|A Q|$ is very small. By symmetry $|A P|=2|A Q|+\left|Q Q^{\prime}\right|$, so if we assume that $|A P|>c>1$ this is impossible. Since $[O, A]$ is outside $T$, and since $\left[A, A^{\prime}\right]$ is perpendicular to $\partial T$ at $A$, we have shown that either $[A, P]$ has uniformly bounded length, or the angle between $[O, A]$ and $[A, P]$ is bounded away from 0 .

It follows in all cases that $[O, A] \cup[A, P]$ is a uniform quasi-geodesic and hence that $d(O, P) \succ R$. The result follows by convexity.

Corollary 5.16. If $\hat{i}_{\infty}(\xi) \notin \mathcal{P}_{\infty}$, then $\hat{i}_{n}(\xi) \rightarrow \hat{i}_{\infty}(\xi)$.

Proof. This follows immediately from Theorem 5.9 .

This completes the proof of Proposition 5.12 .

Remark 5.17. In the proof of Theorem C, we used the pseudo-Anosov $\chi=\left.\alpha\right|_{R}$ only to get a simply degenerate manifold corresponding to a representation of $\pi_{1}(R)$ in the algebraic limit. We could replace the sequence $G_{n}$ with any sequence of representations $\rho_{n}^{\prime}$ of $\pi_{1}(S)$ such that

a) the sequence $\left.\rho_{n}^{\prime}\right|_{\pi_{1}(R)}$ converges to a simply degenerate representation of $\pi_{1}(R)$ and

b) the sequence $\left.\rho_{n}^{\prime}\right|_{\pi_{1}(L)}$ converges to a quasi-Fuchsian representation of $\pi_{1}(L)$.

Then the general form [15] Theorem 5.2, which applies to the case in which the geometry of the limit manifolds do not necessarily have bounded geometry, together with a suitably modified version of Theorem $\mathrm{A}$, would furnish the same conclusion as Theorem $\mathrm{C}$, where we replace the unstable lamination of $\chi$ with the ending lamination of $G_{\infty}$.

\section{REFERENCES}

[1] I. Agol. Tameness of hyperbolic 3-manifolds. arXiv:math.GT/0405568, 2004.

[2] J. Anderson and D. Canary. Algebraic limits of Kleinian groups which rearrange the pages of a book. Invent. Math., 126, 205214, 1996.

[3] J. Anderson and D. Canary. Cores of hyperbolic 3-manifolds and limits of Kleinian groups. Amer. J. Math., 118, 745-779, 1996.

[4] J. Anderson, D. Canary and D. McCullough. On the topology of deformation spaces of Kleinian groups. Ann. of Math., 152, 693-741, 2000.

[5] F. Bonahon. Bouts des variétés de dimension 3. Ann. of Math., 124, 71-158, 1986.

[6] B. H. Bowditch. Relatively hyperbolic groups. Internat. J. Algebra and Computation, 22, 1250016-1 1250016-66, 2012.

[7] B. H. Bowditch. The Cannon-Thurston map for punctured surface groups Math. Z., 255, 35 -76, 2007.

[8] B. H. Bowditch. Stacks of hyperbolic spaces and ends of 3-manifolds. In C. Hodgson et al eds, Geometry and Topology Down Under, Amer. Math. Soc. Contemporary Mathematics Series, to appear. 
[9] J. Brock. Iteration of mapping classes and limits of hyperbolic 3-manifolds. Invent. Math., 1043, 523 $570,2001$.

[10] J. Brock, R. Canary, Y. Minsky. The classification of Kleinian surface groups, II: The Ending Lamination Conjecture. Ann. of Math., 176, 1-142, 2012.

[11] D. Calegari and D. Gabai. Shrinkwrapping and the taming of hyperbolic 3-manifolds. J. Amer. Math. Soc., 19, $38-446,2006$.

[12] R. Canary, D. Epstein and P. Green. Notes on notes of Thurston. In D. Epstein, ed., Analytical and Geometric Aspects of Hyperbolic Space, LMS Lecture Notes 111, 3-92. Cambridge University Press, 1987.

[13] R. Canary, Y. Minsky On limits of tame hyperbolic 3-manifolds. J. Differential Geom., 43, 1-41, 1996.

[14] J. Cannon and W. P. Thurston. Group Invariant Peano Curves. Geom. Topol., 11, 1315-1355, 2007.

[15] S. Das and M. Mj. Addendum to Ending Laminations and Cannon-Thurston Maps: Parabolics. arXiv:1002.2090, 2010.

[16] D. B. A. Epstein and A. Marden. Convex hulls in hyperbolic space, a theorem of Sullivan, and measured pleated surfaces. In D. Epstein, ed., Analytical and Geometric Aspects of Hyperbolic Space, LMS Lecture Notes 111, 3-92. Cambridge University Press, 1987.

[17] R. Evans. Deformation spaces of hyperbolic 3-manifolds: strong convergence and tameness. Ph.D. Thesis, Unversity of Michigan, 2000.

[18] R. Evans. Weakly Type-Preserving Sequences and Strong Convergence. Geometriae Dedicata, 108, 71-92, 2004.

[19] A. Fathi, P. Laudenbach, and V. Poénaru, Travaux de Thurston sur les surfaces, Astérisque 66-67, Société Mathématique de France (1979).

[20] B. Farb. Relatively Hyperbolic groups. Geom. Funct. Anal. , 8, 810-840, 1998.

[21] W. Floyd. Group completions and limit sets of Kleinian groups. Invent. Math., 57, 205-218, 1980.

[22] T. Jørgensen and A. Marden. Algebraic and Geometric convergence of Kleinian groups. Math. Scand., 66, 47-72, 1990.

[23] M. Kapovich. Hyperbolic manifolds and discrete groups. Birkhäuser, 2000.

[24] A. Marden. Outer Circles: An introduction to hyperbolic 3-manifolds. Cambridge University Press, 2007.

[25] M. Mccullough and A. Miller and G. A. Swarup. Uniqueness of cores of noncompact 3-manifolds. J. London Math. Soc. 32, 548-556, 1985.

[26] C. McMullen. Renormalization and 3-manifolds which fiber over the circle. Annals of Math. Studies 142. Princeton University Press, 1996.

[27] Y. N. Minsky. Teichmüller geodesics and ends of hyperbolic 3-manifolds. Topology, 32, 1 - 25, 1992.

[28] Y. N. Minsky. On rigidity, limit sets, and end invariants of hyperbolic 3-manifolds. J. Amer. Math. Soc., $7,539-588,1994$.

[29] Y. N. Minsky. The Classification of Kleinian surface groups I: Models and Bounds. Ann. of Math. , 171, $1-107,2010$.

[30] Y. N. Minsky. End Invariants and the Classification of Hyperbolic 3-manifolds. Current Developments in Mathematics, Vol. 2002, 111 - 141, 2002.

[31] M. Mitra. Cannon-Thurston Maps for Trees of Hyperbolic Metric Spaces. J. Differential Geom., 48,135 $-164,1998$. 
[32] H. Miyachi. Moduli of continuity of Cannon-Thurston maps. In Y. Minsky, M. Sakuma and C. Series eds., Spaces of Kleinian groups, LMS Lecture Notes 329, 121 -150. Cambridge University Press, 2006.

Notes on notes of Thurston. In D. Epstein, ed., Analytical and Geometric Aspects of Hyperbolic Space, LMS Lecture Notes 111, 3-92. Cambridge University Press, 1987.

[33] M. Mj. Ending Laminations and Cannon-Thurston Maps. preprint, arXiv:math.GT/0702162, 2007.

[34] M. Mj. Cannon-Thurston Maps for Pared Manifolds of Bounded Geometry. Geom. Topol. 13, 189-245, 2009.

[35] M. Mj. Cannon-Thurston Maps, i-bounded Geometry and a Theorem of McMullen. Actes du Séminaire de Théorie spectrale et géométrie (Grenoble), 28, 63 - 107, 2009-2010.

[36] M. Mj. Cannon-Thurston Maps and Bounded Geometry. Ramanujan Math. Soc. Lect. Notes Ser. 10, 489 $-511,2010$.

[37] M. Mj. Cannon-Thurston Maps for Kleinian Groups. preprint, arXiv:1002.0996, 2010.

[38] M. Mj and C. Series. Limits of Limit Sets I. Geometriae Dedicata, 1-33, 2012.

[39] M. Mj. Cannon-Thurston Maps for Surface Groups. Ann. of Math., to appear, 2013.

[40] M. Mj and C. Series. Limits of Limit Sets III: The general case. In preparation.

[41] J-P. Otal, Le théorème d'hyperbolisation pour les variétés fibrées de dimension 3, Astérisque 235, Société Mathématique de France, (1996).

[42] W. P. Thurston. The Geometry and Topology of 3-Manifolds. Princeton University Notes, 1980.

[43] W. P. Thurston. Three dimensional manifolds, Kleinian groups and hyperbolic geometry. Bull. Amer. Math. Soc. 50, $357-382,1982$.

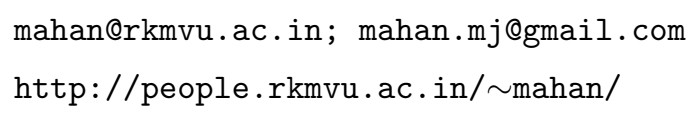

School of Mathematical Sciences, RKM Vivekananda University

P.O. Belur Math, Dt. Howrah, WB 711202, India

\author{
C.M.Series@warwick.ac.uk \\ http://www . maths. warwick. ac.uk/ masbb/
}

Mathematics Institute, University of Warwick

Coventry CV4 7AL, UK 